\title{
Ceres' spectral link to carbonaceous chondrites-Analysis of the dark background materials
}

\author{
Michael SCHÄFER ${ }^{1 *}$, Tanja SCHÄFER ${ }^{2,3}$, Matthew R. M. IZAWA ${ }^{4}$, Edward A. CLOUTIS ${ }^{5}$, \\ Stefan E. SCHRÖDER ${ }^{1}$, Thomas ROATSCH ${ }^{1}$, Frank PREUSKER ${ }^{1}$, Katrin STEPHAN ${ }^{1}$, \\ Klaus-Dieter MATZ ${ }^{1}$, Carol A. RAYMOND ${ }^{6}$, and Christopher T. RUSSELL ${ }^{7}$ \\ ${ }^{1}$ Planetary Geology Department, Institute of Planetary Research, German Aerospace Center (DLR), Rutherfordstr. 2, 12489, \\ Berlin, Germany \\ ${ }^{2}$ Department of Planets and Comets, Max Planck Institute for Solar System Research (MPS), Justus-von-Liebig-Weg 3, 37077, \\ Göttingen, Germany \\ ${ }^{3}$ Department of Geochemistry, Mineralogy and Salt Deposits, Institute of Disposal Research, Clausthal University of \\ Technology, Adolph-Roemer-Str. 2A, 38678, Clausthal-Zellerfeld, Germany \\ ${ }^{4}$ Institute for Planetary Materials, Okayama University, 827 Yamada, Misasa, Tottori 682-0193, Japan \\ ${ }^{5}$ Department of Geography, University of Winnipeg, 515 Portage Avenue, Winnipeg, Manitoba R3B 2E9, Canada \\ ${ }^{6}$ Jet Propulsion Laboratory (JPL), California Institute of Technology, 4800 Oak Grove Drive, Pasadena, California 91109, USA \\ ${ }^{7}$ Institute of Geophysics and Planetary Physics, University of California, Los Angeles (UCLA), Los Angeles, California \\ 90024-1567, USA \\ *Corresponding author. E-mails: m.schaefer@dlr.de, michael.schaefer@signale.de
}

(Received 30 May 2017; revision accepted 01 March 2018)

\begin{abstract}
Ceres' surface has commonly been linked with carbonaceous chondrites (CCs) by ground-based telescopic observations, because of its low albedo, flat to red-sloped spectra in the visible and near-infrared (VIS/NIR) wavelength region, and the absence of distinct absorption bands, though no currently known meteorites provide complete spectral matches to Ceres. Spatially resolved data of the Dawn Framing Camera (FC) reveal a generally dark surface covered with bright spots exhibiting reflectance values several times higher than Ceres' background. In this work, we investigated FC data from High Altitude Mapping Orbit (HAMO) and Ceres eXtended Juling (CXJ) orbit ( 140 m/pixel) for global spectral variations. We found that the cerean surface mainly differs by spectral slope over the whole FC wavelength region $(0.4-1.0 \mu \mathrm{m})$. Areas exhibiting slopes $<-10 \% \mu \mathrm{m}^{-1}$ constitute only $\sim 3 \%$ of the cerean surface and mainly occur in the bright material in and around young craters, whereas slopes $\geq-10 \% \mu^{-1}$ occur on more than $90 \%$ of the cerean surface; the latter being denoted as Ceres' background material in this work. FC and Visible and Infrared Spectrometer (VIR) spectra of this background material were compared to the suite of CCs spectrally investigated so far regarding their VIS/NIR region and $2.7 \mu \mathrm{m}$ absorption, as well as their reflectance at $0.653 \mu \mathrm{m}$. This resulted in a good match to heated CI Ivuna (heated to $200-300{ }^{\circ} \mathrm{C}$ ) and a better match for CM1 meteorites, especially Moapa Valley. This possibly indicates that the alteration of CM2 to CM1 took place on Ceres.
\end{abstract}

\section{INTRODUCTION}

Carbonaceous chondrites (CCs) are the classical material Ceres has been linked with by asteroid taxonomy (Bus and Binzel 2002a, 2002b; DeMeo et al. 2009) and early ground-based observations (Gaffey and McCord 1977; Lebofsky 1978; Larson et al. 1979).
Several other observers found no explicit match to a single CC spectrum (Chapman and Salisbury 1973), but found similarities to $\mathrm{CC}$ constituents such as mixtures of phyllosilicates and opaques (e.g., Johnson and Fanale 1973; Milliken and Rivkin 2009). To date, only the thermally metamorphosed ungrouped chondrites Y86720 and Y-82162 have been associated with Ceres and 
other C-type asteroids (Hiroi et al. 1993, 1994). These spectral matches are based on their neutral to red spectral slopes (with the term "red slope" denoting increasing reflectance with increasing wavelength), a shallow broad absorption feature ranging approximately from 0.8 to $1.5 \mu \mathrm{m}$, and a lack of strong absorption features over the $0.4-2.5 \mu \mathrm{m}$ range. Some earlier ground-based observations of Ceres in the visible region describe a faint absorption between 0.6 and $0.7 \mu \mathrm{m}$ assigned to iron in hydrated minerals (Vilas and McFadden 1992; Vilas et al. 1993; Fornasier et al. 1999; Perna et al. 2015), which was not detected in the large asteroid surveys (Bus and Binzel 2002b; Lazarro et al. 2004). Further ground-based observations in the mid infrared detected an absorption feature between 3.0 and $3.1 \mu \mathrm{m}$ that was first assigned to structural $\mathrm{OH}$-groups and interlayer water or water ice in clay minerals (Lebofsky et al. 1981). Later, higher spectral resolution observations of this feature were attributed to brucite (Milliken and Rivkin 2009), ammoniated clays (King et al. 1992), organics plus water ice (Vernazza et al. 2005), or carboxylic acids (Applin et al. 2016), none of which have been uniquely detected in CC spectra to date.

In March 2015, NASA's Dawn spacecraft started acquisition of multispectral image data with the onboard Framing Camera (FC; Sierks et al. 2011) and the Visible and Infrared Spectrometer (VIR; De Sanctis et al. 2011). Observations from Dawn FC generally show a dark surface covered with bright spots exhibiting reflectances up to several times higher than the dark background (Nathues et al. 2015). The average spectrum of Ceres from Dawn VIR (De Sanctis et al. 2015) exhibits an OH-absorption due to phyllosilicates at $2.7 \mu \mathrm{m}$ (De Sanctis et al. 2015; Ammannito et al. 2016), absorptions between 3.0 and $3.1 \mu \mathrm{m}$ assigned to ammoniated phyllosilicates, a carbonate and/or organics absorption at $\sim 3.4 \mu \mathrm{m}$, and a further carbonate absorption at $3.9 \mu \mathrm{m}$.

In our work, we use the visible and near-infrared (VIS/NIR) wavelength region covered by the FC instrument $(0.4-1.0 \mu \mathrm{m})$ to detect and characterize Ceres' dark background material in the context of a large number of CCs spectrally investigated so far. Additionally, we use the $2.7 \mu \mathrm{m}$ absorption covered by the VIR instrument, which is appropriate to characterize the phyllosilicates of $\mathrm{CCs}$ as shown by Beck et al. (2010) and Takir et al. (2013). Schäfer et al. (2016) have shown that CCs can largely be distinguished from each other by two spectral ratios using FC filters. The discrimination scheme by Schäfer et al. (2016) is based on the spectral slope in FC's short-wavelength region toward the UV, and on the slope in the NIR region of the FC (i.e., wavelengths up to $1 \mu \mathrm{m})$.

\section{DATA AND METHODS}

\section{Framing Camera Image Products}

The Dawn FC acquires images utilizing seven color filters with effective wavelengths of $0.438,0.555,0.653$, $0.749,0.829,0.917$, and $0.965 \mu \mathrm{m}$, plus one panchromatic filter (termed "clear filter"). The FC acquired several spectral data sets of Ceres with increasing spatial resolution up to $\sim 35 \mathrm{~m} /$ pixel during the Low Altitude Mapping Orbit (LAMO) phase. Since during LAMO no complete spatial coverage of color data was gathered, for this work we use FC data from the preceding High Altitude Mapping Orbit (HAMO) phase with a resolution of $\sim 140 \mathrm{~m} /$ pixel. However, during the HAMO phase, the southern high latitudes of Ceres (approximately south of $55^{\circ} \mathrm{S}$ ) were acquired using only four FC color filters. Fortunately, during Dawn's extended mission after LAMO, termed Ceres eXtended Juling orbit (CXJ), the south pole has been acquired with all color filters at a spatial resolution $(\sim 160 \mathrm{~m} /$ pixel) comparable to HAMO. For this reason, we use a combined HAMO and CXJ color filter mosaic for this work (spatial resolution resampled to $140 \mathrm{~m} /$ pixel).

The raw, level 1A, FC color filter images were calibrated and processed using the image processing software VICAR developed by NASA/JPL. Data calibration was done as described in Schröder et al. (2013, 2014), including correction of stray light, yielding reflectance data (radiance factor, I/F). Data processing includes image ortho-rectification to map projection, photometric correction to standard viewing geometry $\left(30^{\circ}\right.$ incidence, $0^{\circ}$ emission, $30^{\circ}$ phase angle), and generation of controlled global image mosaics (Roatsch et al. 2016). In this paper, we generally refer to the photometrically corrected reflectance as the "absolute I/ $\mathrm{F}$," or as "normalized $\mathrm{I} / \mathrm{F}$ " in cases where the reflectances are normalized to one of the filters. Photometric correction was performed using the Akimov disk function in combination with polynomial phase functions (for coefficients, see table 5 in Schröder et al. 2017), utilizing a HAMO shape model produced by stereophotogrammetry (Preusker et al. 2016; see also Fig. 1b). We note that there is still insufficient knowledge about photometric properties of the cerean surface, especially for high-phase angles when using the FC color filters, since the Akimov phase function parameters in Schröder et al. (2017) are best valid for phase angles between $7^{\circ}$ and $49^{\circ}$. Therefore, larger uncertainties in absolute reflectance spectra are associated with areas that were imaged at high incidence angle, which are predominantly found in high-latitude regions. 


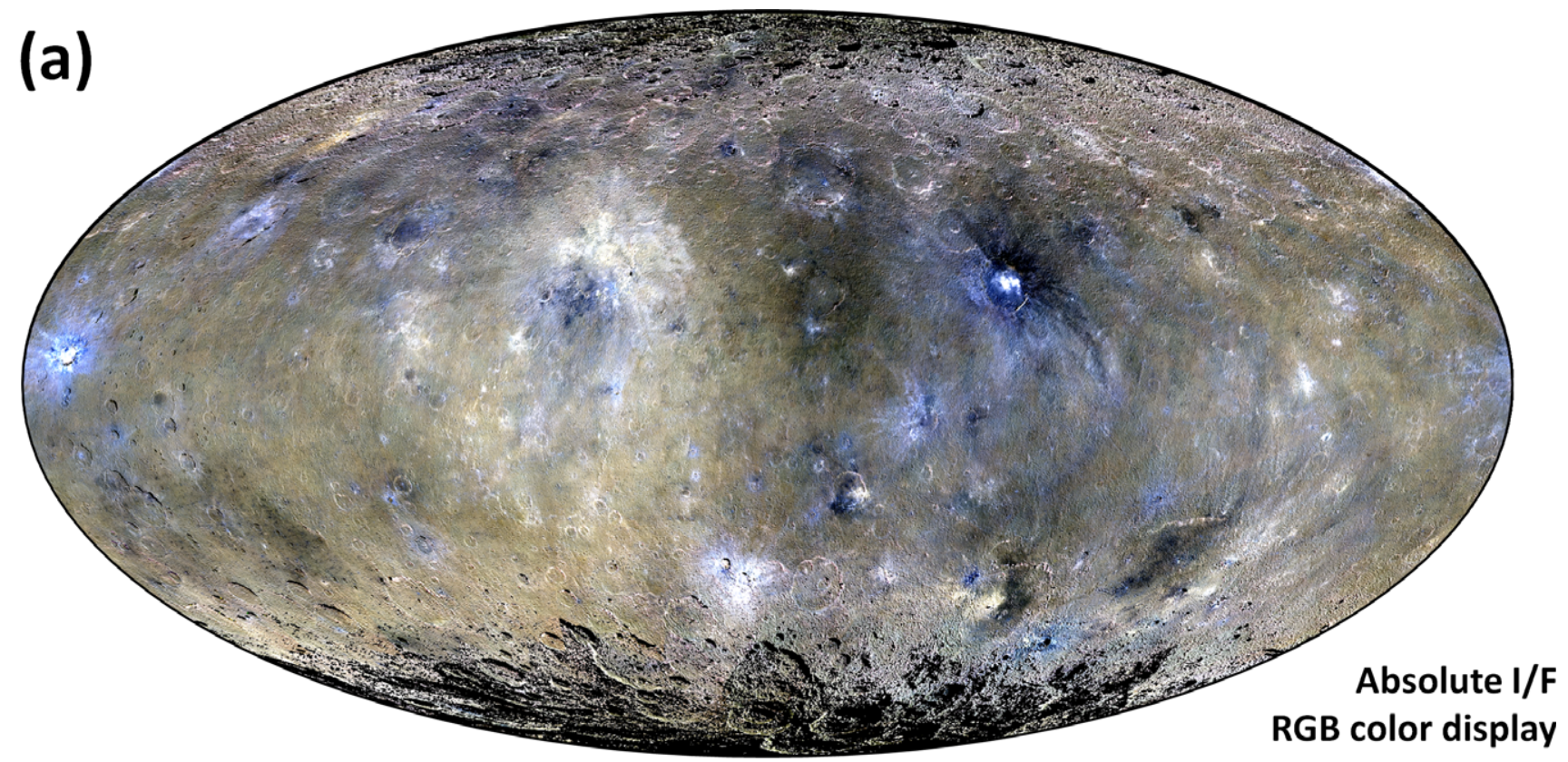

$\square R_{0.965 \mu m}(3.0-3.8 \%) \quad \square R_{0.749 \mu m}(3.1-4.0 \%) \quad \square R_{0.438 \mu m}(3.0-3.8 \%)$

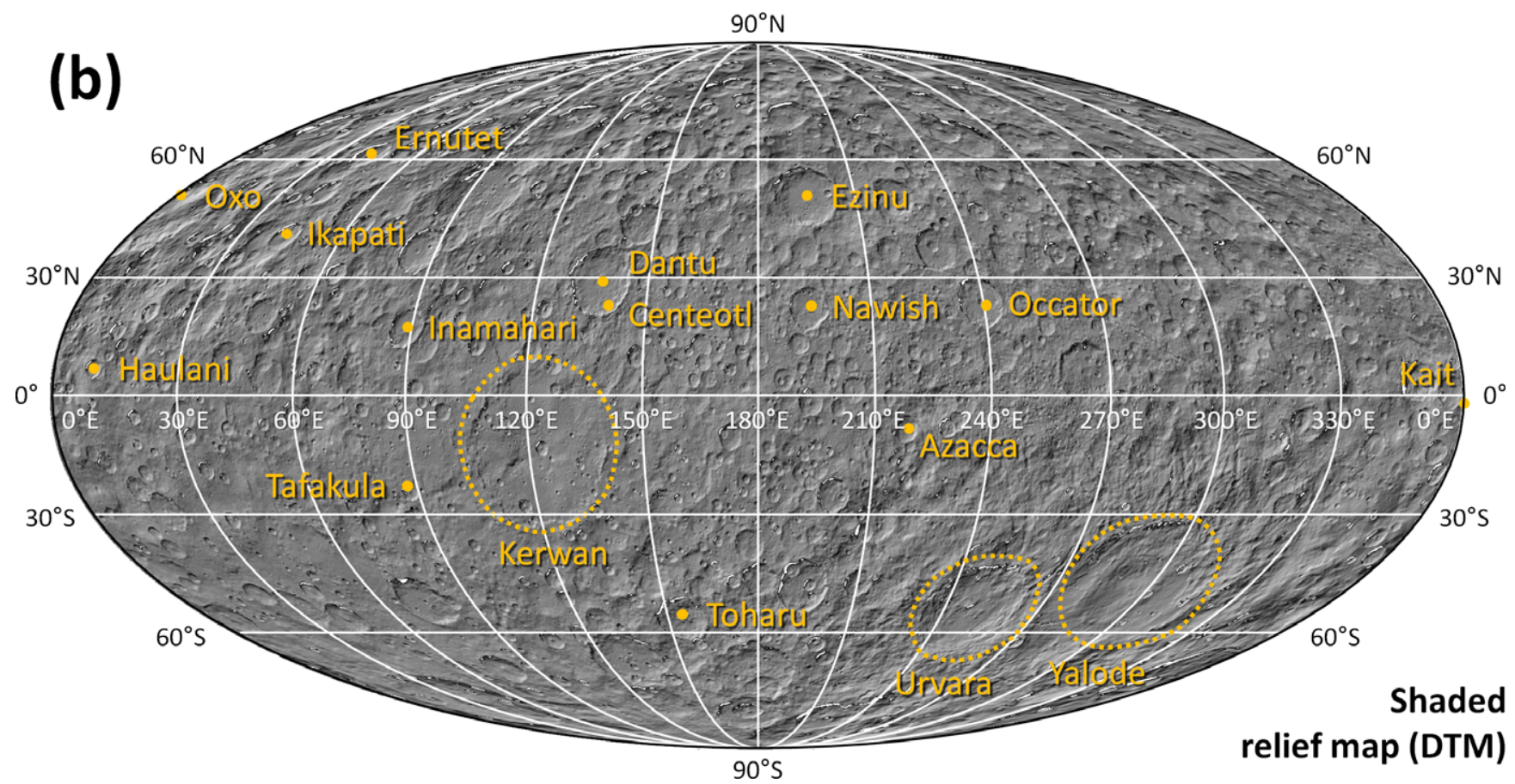

Fig. 1. Global maps of Ceres based on Dawn FC HAMO data in equal-area "Mollweide" projection. a) Photometrically corrected $\mathrm{I} / \mathrm{F}$ reflectances displayed in RGB color space $\left(R=R_{0.965 \mu \mathrm{m}}, G=R_{0.749 \mu \mathrm{m}}, B=R_{0.438 \mu \mathrm{m}}\right)$, showing differences in absolute reflectance and relative differences between color filters. No spectral information about black areas near the poles is available due to high phase angle conditions or shadows. b) Shaded relief map of digital terrain model from HAMO orbit, overlaid by coordinates and crater names mentioned in the text (basins are encircled with dotted lines).

In addition to the calibration described in Schröder et al. $(2013,2014)$, we made small modifications to the absolute $\mathrm{I} / \mathrm{F}$ in order to diminish the magnitude of the existing stray light correction which influences the absolute I/F level of each FC filter (see Supplement A in supporting information for further information and 
Table A1 for correction values). This additional correction yields in $\mathrm{I} / \mathrm{F}$ levels comparable to early Dawn FC observations from Rotation Characterization orbit 1 (RC1) where this additional stray light influence is negligible. Furthermore, we suspect that filter F2 at $0.555 \mu \mathrm{m}$ shows too high reflectance values for reasons that are still unknown (see Supplement B in supporting information for further information). Therefore, we excluded filter F2 from further investigations or spectral comparisons in this work.

In order to determine accurate and comparable averaged spectra or spectral metrics of differently sized areas, we present all FC mosaics and derived maps in Mollweide projection. This equal-area map projection allows comparing proportions in area, since all pixels show an equal area of Ceres, independent of latitude.

For comparison with laboratory spectra of CC meteorites (see also the Laboratory Spectra of Meteorites section), Schäfer et al. (2016) used the two spectral ratios, $R_{0.555 \mu \mathrm{m}} / R_{0.438 \mu \mathrm{m}}\left(S_{\mathrm{VIS}}\right)$ and $R_{0.829 \mu \mathrm{m}} /$ $R_{0.965 \mu \mathrm{m}}\left(S_{\mathrm{NIR}}\right)$. As a result of the abovementioned defect in FC filter F2 at $0.555 \mu \mathrm{m}$, we substituted $S_{\mathrm{VIS}}$ by the ratio $R_{0.653 \mu \mathrm{m}} / R_{0.438 \mu \mathrm{m}}$ and obtained slightly differing but still valid $\mathrm{CC}$ discrimination fields for the major CC groups compared to those in Schäfer et al. (2016) (see the Spectral Comparison to CC Meteorites section). For these changes, we show in Supplement D in supporting information the new $\mathrm{CC}$ discrimination scheme including all meteorites that the derived field outlines are based on (cf. Laboratory Spectra of Meteorites section and Table E1 in Supplement E in supporting information).

For investigating cerean surface variability, we used the slope of the spectrum across the full range of FC bands (wavelength range $0.4-1.0 \mu \mathrm{m}$ ) as a key parameter (in the following denoted with the Greek symbol $\gamma$ ). This was done pixel-by-pixel by determining the linear regression (or linear fit) through the normalized $\mathrm{I} / \mathrm{F}$ values of all color filters except $\mathrm{F} 2$ (normalized to $0.653 \mu \mathrm{m}$ ) using the routine POLY_FIT with degree one of the programming language Interactive Data Language (IDL). The slope has units of percent reflectance per micrometer $\left(\% \mu \mathrm{m}^{-1}\right)$. This approach is advantageous compared to the use of a simple reflectance ratio (e.g., $R_{0.438 \mu \mathrm{m}} / R_{0.965 \mu \mathrm{m}}$ ), since the slope includes many FC color filters and is thus less dependent on calibration errors in single filters. Please note that in the center of the brightest faculae within crater Occator (center coordinates $20^{\circ} \mathrm{N} / 239^{\circ} \mathrm{E}$ ), derived spectral parameters are in some places inaccurate due to partial saturation of the FC color filter images. Moreover, the used photometric correction with global uniform parameters is inaccurate for the faculae as their photometric properties are different from Ceres average (Schröder et al. 2017).

The Dawn data can be obtained from the NASA Planetary Data System ${ }^{1}$ (PDS). All coordinates used in this paper are based on the "Kait system" (Roatsch et al. 2015) defined by the longitude of crater Kait (center coordinates $2^{\circ} \mathrm{S} / 0^{\circ} \mathrm{E}$ ). The topographic names and center coordinates of craters used in this paper are approved by the International Astronomical Union (IAU) Working Group for Planetary System Nomenclature ${ }^{2}$ (WGPSN; version as of August 25, 2017).

\section{VIR Imaging Spectrometer Data}

The Dawn VIR instrument consists of two spectrometers, one for the spectral range between 0.25 and $1.07 \mu \mathrm{m}$ (VIR-VIS), and one for the range between 1.02 and $5.10 \mu \mathrm{m}$ (VIR-IR). Due to a missing calibration, VIR-VIS data may not be used shortward of $\sim 0.45 \mu \mathrm{m}$ (communicated within the Dawn Science Team by Andrea Raponi in 2015).

We used level 1C data from VIR-IR, in which sensor noise has been slightly suppressed using a spectral boxcar average filter. In addition, we applied calibration correction factors provided by the VIR team on October 25, 2015. We used ISIS 3 for the data processing to $\mathrm{I} / \mathrm{F}$ data, photometric correction using the Lommel-Seeliger function, and reprojection to map coordinates. Due to the use of this very simple photometric function, errors in absolute reflectance values are likely. However, this is tolerable for our purposes, since we used VIR data to show the general spectral shape and characteristics like absorption band centers and not for quantitative measurements. Finally, after extraction of average spectra for the two regions of interest using the software ENVI, a simple thermal correction of the IR spectra was performed in Excel based on Planck's law, by removing the thermal flux of a black body possessing an assumed temperature, which was manually determined.

In our study, we used two VIR-IR scenes from the Survey phase possessing a spatial resolution of $\sim 1100 \mathrm{~m} /$ pixel. In VIR scene \#487410275, we extracted an averaged spectrum of a region of interest (ROI) approximately $90 \times 40 \mathrm{~km}$ in size, located $\sim 20 \mathrm{~km}$ west of crater Tafakula (within the "orange medium region"; center coordinates $20^{\circ} \mathrm{S} / 89^{\circ} \mathrm{E}$; see also map in Fig. 1b). In VIR scene \#487375659, we extracted an averaged spectrum of a ROI approximately $20 \times 85 \mathrm{~km}$ in size directly adjacent to the eastern rim of crater Ikapati (within the "yellow medium region"; $34^{\circ} \mathrm{N} / 46^{\circ} \mathrm{E}$ ).

\footnotetext{
${ }^{1}$ http://pds.nasa.gov/ and https://sbn.psi.edu/pds/resource/dwncfc2.html ${ }^{2}$ https://planetarynames.wr.usgs.gov/
} 


\section{Laboratory Spectra of Meteorites}

We use a CC discrimination scheme with individual fields for each major CC group which is based on 121 CC laboratory spectra (all meteorite classifications are verified by the Meteoritical Bulletin Database of the Meteoritical Society ${ }^{3}$; for details on the used meteorite spectra, see Schäfer et al. 2016); they were compiled from the Reflectance Experiment Laboratory ${ }^{4}$ (RELAB) database at Brown University, USA. In order to select comparable spectra, we only used spectra of powders with grain sizes ranging from $<75 \mu \mathrm{m}$ to $<125 \mu \mathrm{m}$, i.e., including all the fines. All meteorites used in this study are listed in Table E1 in Supplement E. Additionally, we used spectra from the Planetary Spectrophotometer Facility ${ }^{5}$ at the University of Winnipeg, Canada, for the investigation of grain size effects (Izawa et al. 2016). The spectra were resampled to Dawn FC band passes using filter response functions (using filter transmission profiles; cf. fig. 36 in Sierks et al. 2011) in the software ENVI.

The discrimination diagram (cf. Supplement D) is based on two spectral ratios incorporating four FC filters (see the Framing Camera Image Products section). It enables discrimination of spectra between the major $\mathrm{CC}$ groups $(\mathrm{CM}, \mathrm{CR}, \mathrm{CV}, \mathrm{CO}$, and $\mathrm{CK})$ with only minor overlap between groups. Schäfer et al. (2016) developed very similar discrimination fields; however, the fields in this work exhibit a slightly changed shape, because we used FC filter F7 at $0.653 \mu \mathrm{m}$ instead of filter F2 at $0.555 \mu \mathrm{m}$ (see the Framing Camera Image Products section).

In this work, we extend our investigations by including artificially heated CI Ivuna ${ }^{6}$ from Hiroi et al. (1996) and artificially heated CM Murchison ${ }^{7}$ from Hiroi et al. (1994). Furthermore, we upgraded the discrimination fields of Schäfer et al. (2016) by enclosing five aqueously altered and thermally metamorphosed carbonaceous chondrites (ATCC) in a new field termed "Belgica-7904-like." This field encompasses the meteorites Belgica $7904,{ }^{8}$ Dhofar 225, ${ }^{9}$ Dhofar $735,{ }^{10}$ Y-82162, ${ }^{11}$ and Y-86720 $0^{12}$ according to criteria summarized in Ivanova et al. (2010), which include their oxygen isotopic composition (high ${ }^{18} \mathrm{O}$

\footnotetext{
${ }^{3}$ http://www.lpi.usra.edu/meteor/metbull.php

${ }^{4}$ http://www.planetary.brown.edu/relab/

${ }^{5}$ http://psf.uwinnipeg.ca/

${ }^{6}$ https://www.lpi.usra.edu/meteor/metbull.php?code $=12063$

${ }^{7}$ https://www.lpi.usra.edu/meteor/metbull.php?code $=16875$

${ }^{8}$ https: //www.lpi.usra.edu/meteor $/$ metbull.php? $\operatorname{code}=5001$

${ }^{9}$ https://www.lpi.usra.edu/meteor $/$ metbull.php?code $=7009$

${ }^{10}$ https://www.lpi.usra.edu/meteor/metbull.php?code $=7488$

${ }^{11}$ https://www.lpi.usra.edu/meteor/metbull.php?code $=29356$

${ }^{12} \mathrm{https}$ ://www.lpi.usra.edu/meteor/metbull.php?code $=30226$
}

range different from other $\mathrm{CM}$ chondrites), their low $\mathrm{H}_{2} \mathrm{O}$ content in comparison to $\mathrm{CMs}$, and on their partial transformation of phyllosilicates to olivine.

Additionally, for comparison with VIR data in the $3 \mu \mathrm{m}$ wavelength region, we used $\mathrm{CI}$ and $\mathrm{CM}$ spectra from RELAB measured in dry air, and spectra from Takir et al. (2013) measured under vacuum conditions. From the latter ones, adsorbed water was removed by heating the samples to $475 \mathrm{~K}$ prior to the spectral measurements.

\section{RESULTS}

\section{Spectral Diversity of Cerean Surface Material}

FC data from Dawn's approach at Ceres showed spectral diversity of the surface material in different localities on Ceres. Figure 1a uses a simple RGB display, initially used in Nathues et al. (2015), in which the $\mathrm{I} / \mathrm{F}$ reflectance values in the $0.965 \mu \mathrm{m}, 0.749 \mu \mathrm{m}$, and $0.438 \mu \mathrm{m}$ FC filters are used for RGB, respectively. A very similar display, but using the $0.555 \mu \mathrm{m}$ filter instead of the $0.749 \mu \mathrm{m}$ filter in the green channel, is shown in Schröder et al. (2017) based on Dawn FC data from Rotation Characterization orbit 3 (RC3). In Fig. 1a, predominantly brownish surface areas are visible, plus scattered smaller areas of bluish colors. Bluish areas are commonly associated with impact craters, e.g., Haulani (center coordinates $6^{\circ} \mathrm{N} / 11^{\circ} \mathrm{E}$ ) and Occator (see Fig. 1b for a shaded relief map with place names and coordinates).

The main difference between the spectra of brownish and bluish material is their spectral slope, where blue slopes stand for a decrease of $\mathrm{I} / \mathrm{F}$ with increasing wavelength. In this work, we directly use the spectral slope $\gamma$ (in percent reflectance per micrometer) over the whole FC spectral range $(0.4-1.0 \mu \mathrm{m}$, except filter F2), as described in the Framing Camera Image Products section. This spectral slope is displayed in Fig. 2a with high slope values (positive; red spectral slopes) shown in bright tones of gray or white, while negative values (blue spectral slopes) are shown in dark gray to black tones. Note that the lowest slope values occur at Haulani and Oxo $\left(42^{\circ} \mathrm{N} / 0^{\circ} \mathrm{E}\right)$, while Occator rather reveals less bluish slopes (also observed by Schröder et al. 2017). Aside from these rather tiny regions, the prevailing majority of the surface of Ceres appears neutral to slightly red sloped. A quite similar display as in Fig. 2a, but based on the ratio $R_{0.965 \mu \mathrm{m}} /$ $R_{0.438 \mu \mathrm{m}}$ derived from RC3 data, was published in Schröder et al. (2017). Stephan et al. (2017) used the spectral ratio $R_{0.438 \mu \mathrm{m}} / R_{0.749 \mu \mathrm{m}}$ in particular to highlight bluish regions, which show good accordance with the dark regions in Fig. 2a. 

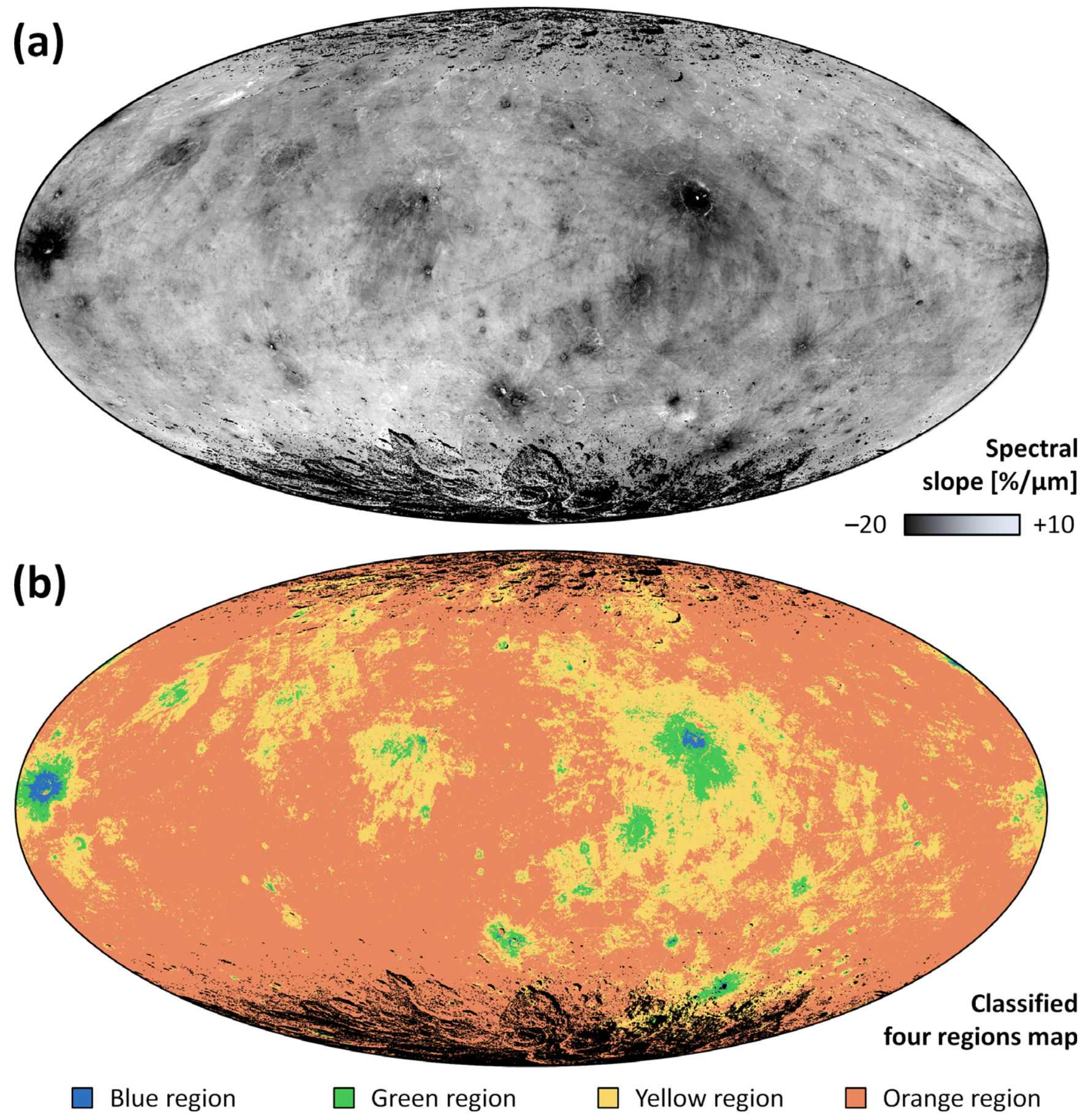

Fig. 2. Global maps of Ceres based on Dawn FC HAMO data in equal-area "Mollweide" projection. a) Spectral slope encompassing the whole FC wavelength range $(0.4-1.0 \mu \mathrm{m})$ in percent reflectance per micrometer (positive values indicate spectrally red-sloped, negative values indicate blue-sloped areas). b) Classification map based on spectral slope data, showing four classes of surface units (with increasing spectral slope: blue, green, yellow, and orange) (see Table 1 for classification criteria). No spectral information about black areas near the poles is available due to high phase angle conditions or shadows.

Based on these observations, we subjectively divided the slope values occurring on Ceres into four classes in order to classify and map different surface regions (for a discussion on slope thresholds, see the Spectral Variability of Ceres section). The different slope criteria for each of the four classes are given in Table 1 and 
Table 1. Criteria for classification of different regions and information on respective area sizes found on Ceres. Positive slope values correspond to a red spectral slope over the whole FC wavelength range $(0.4-1.0 \mu \mathrm{m})$, while negative values correspond to bluish spectral slopes.

\begin{tabular}{llc}
\hline Classification designation & Criteria for spectral slope $\gamma(\%$ reflectance per $\mu \mathrm{m})$ & Areal occurrence on Ceres $(\%)$ \\
\hline Orange region & $-3.0 \leq \gamma$ & 67.17 \\
Yellow region & $-10.0 \leq \gamma<-3.0$ & 23.04 \\
Green region & $-20.0 \leq \gamma<-10.0$ & 2.62 \\
Blue region & $\gamma<-20.0$ & 0.26 \\
Shadowed regions & No spectral data available & 6.91 \\
\hline
\end{tabular}

were chosen based on the histogram of the slope values (see Fig. $\mathrm{C} 1$ in Supplement $\mathrm{C}$ in supporting information) in order to derive a meaningful map display. Figure $2 \mathrm{~b}$ exhibits the spatial distribution of the four classes on Ceres. No spectral information is available for high incident regions or shadowed regions, shown as black areas near Ceres' poles. Table 1 lists the areal occurrence of the four classes in percent of the whole cerean surface.

Based on the classified regions shown in Fig. 2b, we derived averaged spectra for all pixels within a specific region. Figure 3 shows the averaged spectra of the orange, yellow, green, and blue regions. The blue spectrum represents the most blue-sloped regions $\left(\gamma<-20 \% \mu \mathrm{m}^{-1}\right)$ in and around Haulani, Oxo, and at a few small spots on the floor of Occator, plus the $5 \mathrm{~km}$ diameter crater Centeotl (center coordinates $19^{\circ} \mathrm{N} /$ $\left.141^{\circ} \mathrm{E}\right)$ on the SE floor of Dantu $\left(24^{\circ} \mathrm{N} / 138^{\circ} \mathrm{E}\right)$. The lowest slope values occur localized at Haulani $\left(-48 \% \mu \mathrm{m}^{-1}\right)$ and Oxo $\left(-42 \% \mu \mathrm{m}^{-1}\right)$, while in Occator and Centeotl, values go down to -35 and $-33 \% \mu \mathrm{m}^{-1}$, respectively. The average slope value of the whole blue region aggregates to $-24.42 \% \mu \mathrm{m}^{-1}$ and predominantly includes bright pixels with the highest absolute $\mathrm{I} / \mathrm{F}$ values on Ceres (Fig. 3a).

The green spectrum represents all pixels with bluish slopes between -20 and $-10 \% \mu \mathrm{m}^{-1}$, which occur, for example, within and around craters Occator, Ikapati, Dantu, Toharu $\left(48^{\circ} \mathrm{S} / 156^{\circ} \mathrm{E}\right)$, or Azacca $\left(7^{\circ} \mathrm{S} / 218^{\circ} \mathrm{E}\right)$. The slope of the whole green region averages to $-12.72 \% \mu \mathrm{m}^{-1}$.

Often, the blue-sloped craters are surrounded by wide areas of lesser sloped material (between -10 and $-3 \% \mu \mathrm{m}^{-1}$ ) which are classified by us as yellow region. The yellow region nearly represents one quarter of the cerean surface, whose average spectrum with a spectral slope of $-5.32 \% \mu \mathrm{m}^{-1}$ is plotted as a yellow line in Fig. 3. Areas with neutral to red spectral slopes (greater equal $-3 \% \mu \mathrm{m}^{-1}$ ) are classified as orange region. This arbitrarily defined area represents two-thirds of the cerean surface. Its average spectrum is depicted in Fig. 3 as an orange spectrum. The average spectral slope of the orange region is $+0.57 \% \mu \mathrm{m}^{-1}$. The steepest red slope values on Ceres (up to $75 \% \mu \mathrm{m}^{-1}$ ) occur at crater Ernutet $\left(53^{\circ} \mathrm{N} / 46^{\circ} \mathrm{E}\right)$. There, a distinct material emerges on Ceres, which is interpreted as aliphatic organic-rich material (De Sanctis et al. 2017; Pieters et al. 2017). Several tiny patches of this material can be found in the vicinity of Ernutet up to $140 \mathrm{~km}$ away from the crater's rim (Pieters et al. 2017). One further small occurrence of the red material was found $\sim 400 \mathrm{~km} \mathrm{SE}$ of Ernutet at crater Inamahari $\left(14^{\circ} \mathrm{N} / 89^{\circ} \mathrm{E}\right.$; De Sanctis et al. 2017). Although this material can be classified by its high spectral slope values (e.g., $\gamma>20 \% \mu \mathrm{m}^{-1}$ ), we did not include it as a discrete class. Its spatial occurrence is negligible $(\sim 0.01 \%$ of Ceres' surface area) and thus is invisible in our maps; additionally, it does not statistically contribute to the average spectrum of the orange class.

Figure 4a displays Ceres' $\mathrm{I} / \mathrm{F}$ reflectance in the $\mathrm{FC}$ $0.653 \mu \mathrm{m}$ filter. When compared to Fig. 2, there are variations in reflectance within the yellow and orange classified regions. In the following, we investigated whether the spectra differ depending on their absolute reflectance. For this reason, we classified further subregions of both the orange and yellow classes by their absolute reflectance at $0.653 \mu \mathrm{m}\left(R_{0.653 \mu \mathrm{m}}\right)$, namely "bright," "medium," and "dark." The reflectance thresholds (see Table 2) were chosen based on the histogram of all reflectance values (see Fig. C2 in Supplement C), in order that the majority of pixels are classified in the medium reflectance classes. Thus, the bright and dark regions, constituting the minority of pixels, can be included in our investigations regarding their spectral deviations from the medium classes. The thresholds for the spectral slope were not changed (see Table 1). Figure $4 b$ shows the map resulting from the classification based on both spectral slope and absolute reflectance. The largest bright regions $\left(R_{0.653 \mu \mathrm{m}} \geq 3.8 \%\right)$ are within and in the vicinity of craters Kerwan (center coordinates $\left.11^{\circ} \mathrm{S} / 124^{\circ} \mathrm{E}\right)$, Dantu, Urvara $\left(46^{\circ} \mathrm{S} / 249^{\circ} \mathrm{E}\right)$, and Inamahari. The largest dark regions $\left(R_{0.653 \mu \mathrm{m}}<3.4 \%\right)$ occur NE of Occator, within and north of basins Urvara and Yalode $\left(43^{\circ} \mathrm{S} / 292^{\circ} \mathrm{E}\right)$, as well as within and in the surrounding of craters Ezinu $\left(43^{\circ} \mathrm{N} / 196^{\circ} \mathrm{E}\right)$ and Nawish $\left(18^{\circ} \mathrm{N} / 194^{\circ} \mathrm{E}\right)$. 

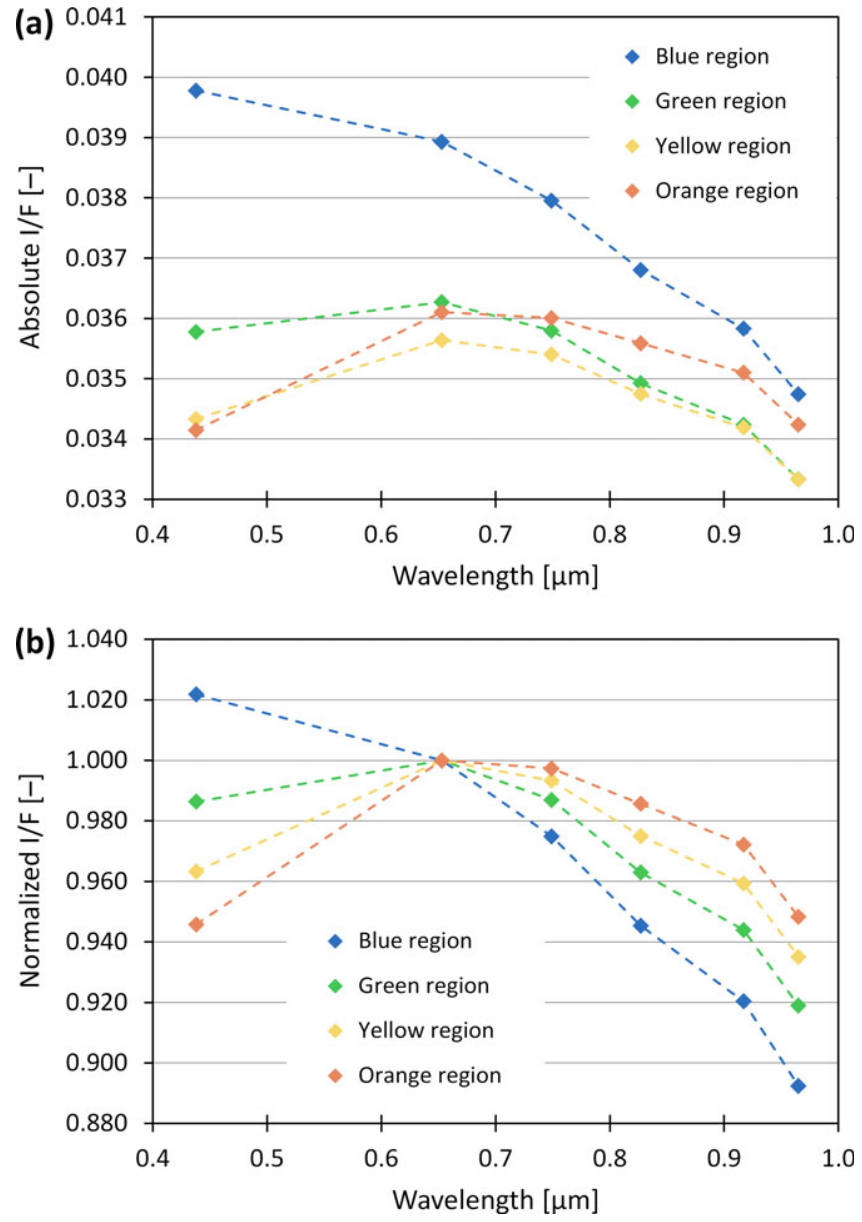

Fig. 3. Average FC color filter spectra of each classified region (same colors as shown in the map in Fig. 2b). a) Absolute $\mathrm{I} / \mathrm{F}$ values. b) $\mathrm{I} / \mathrm{F}$ values normalized at $0.653 \mu \mathrm{m}$.

Averaged spectra of the six newly classified subregions are plotted in Fig. 5. Although hardly recognizable in Fig. 5, the calculated values for the spectral slope of the orange regions increase (redden) very slightly with increasing reflectance, starting from $0.02 \% \mu \mathrm{m}^{-1}$ (orange dark region), over $0.58 \% \mu \mathrm{m}^{-1}$ (orange medium region) to $1.04 \% \mu^{-1}$ (orange bright region). The spectral slope values of the yellow subregions show no dependence with absolute reflectance (yellow dark region, $-5.37 \% \mu \mathrm{m}^{-1}$; yellow medium region, $-5.31 \% \mu \mathrm{m}^{-1}$; and yellow bright region, $-5.34 \% \mu \mathrm{m}^{-1}$ ).

Additionally, we analyzed the FC color mosaics for absorptions at 0.7 and $0.9 \mu \mathrm{m}$ indicative of Fe-bearing phyllosilicates (King and Clark 1989; Calvin and King 1997; Cloutis et al. 2012) by applying spectral parameters developed by Schäfer et al. (2016), but found no evidence for these bands.

\section{Spectral Comparison to CC Meteorites}

For comparison with $\mathrm{CC}$ meteorites, we applied a similar spectral discrimination diagram as the one introduced by Schäfer et al. (2016) for the Dawn FC data (see the sections Framing Camera Image Products and Laboratory Spectra of Meteorites). Figure 6 displays the discrimination fields for major CC groups, as well as ATCCs (see Fig. 6a) and heating pathways of artificially heated Murchison and Ivuna in the same context (Fig. 6b). The orange and yellow point clouds correspond to all pixels of the classified surface units from the FC color mosaic as shown in the Spectral Diversity of Cerean Surface Material section. As can be seen in Fig. 6a, the centers of the point clouds (depicted by diamond symbols) plot outside the fields of the major $\mathrm{CC}$ groups, mainly covering a field of five ATCCs that were described as Belgica-7904-like CCs by Ivanova et al. (2010). Among those Belgica-7904-like ATCCs, two specimens are located nearest to Ceres' point clouds, namely Y-82162 (plotting close to the center of the yellow point cloud), and Dhofar 225 (plotting within the orange point cloud).

Figure $6 \mathrm{~b}$ displays spectral pathways of heating experiments of Ivuna (Hiroi et al. 1996) and Murchison (Hiroi et al. 1994). The Ivuna samples heated from $200{ }^{\circ} \mathrm{C}$ up to $600{ }^{\circ} \mathrm{C}$ also approximate the orange point cloud with Ivuna heated to $200{ }^{\circ} \mathrm{C}$ being closest (Fig. 6b). From the heated Murchison samples, only the one heated to $1000{ }^{\circ} \mathrm{C}$ touches the lower edge of the point clouds. Figure $6 \mathrm{~b}$ also shows the position of two other CIs that were spectrally measured, Orguei ${ }^{13}$ and Alais $^{14}$ (no separate CI field was generated due to the small number of specimens). Please also note that one $\mathrm{CV}$ and a few CK meteorites also plot near Ceres' point clouds (see Fig. D1 in Supplement D); however, CV and $\mathrm{CK}$ meteorites exhibit much more pronounced olivine absorption bands at $1 \mu \mathrm{m}$ and have a much higher reflectance than average Ceres in the VIS.

Figure 6c depicts grain size effects based on a Murchison sample with varying grain size from $<45$ to $<1000 \mu \mathrm{m}$ and grain size fractions including more than $95 \%$ coarse material experimentally determined by Izawa et al. (2016). With coarser grain size, the Murchison spectra get more blue sloped causing a shift in direction to the upper left corner of our parameter space (indicated by a red arrow in Fig. 6c). If we assume the unlikely case (see the Comparison to Meteorites and Origins of Observed Features section) that Ceres' averaged spectra are influenced by grain sizes $<500 \mu \mathrm{m}$, there are nine specimens which could

\footnotetext{
${ }^{13}$ https://www.lpi.usra.edu/meteor/metbull.php?code $=18026$

${ }^{14}$ https://www.lpi.usra.edu/meteor $/$ metbull.php? $\operatorname{code}=448$
} 

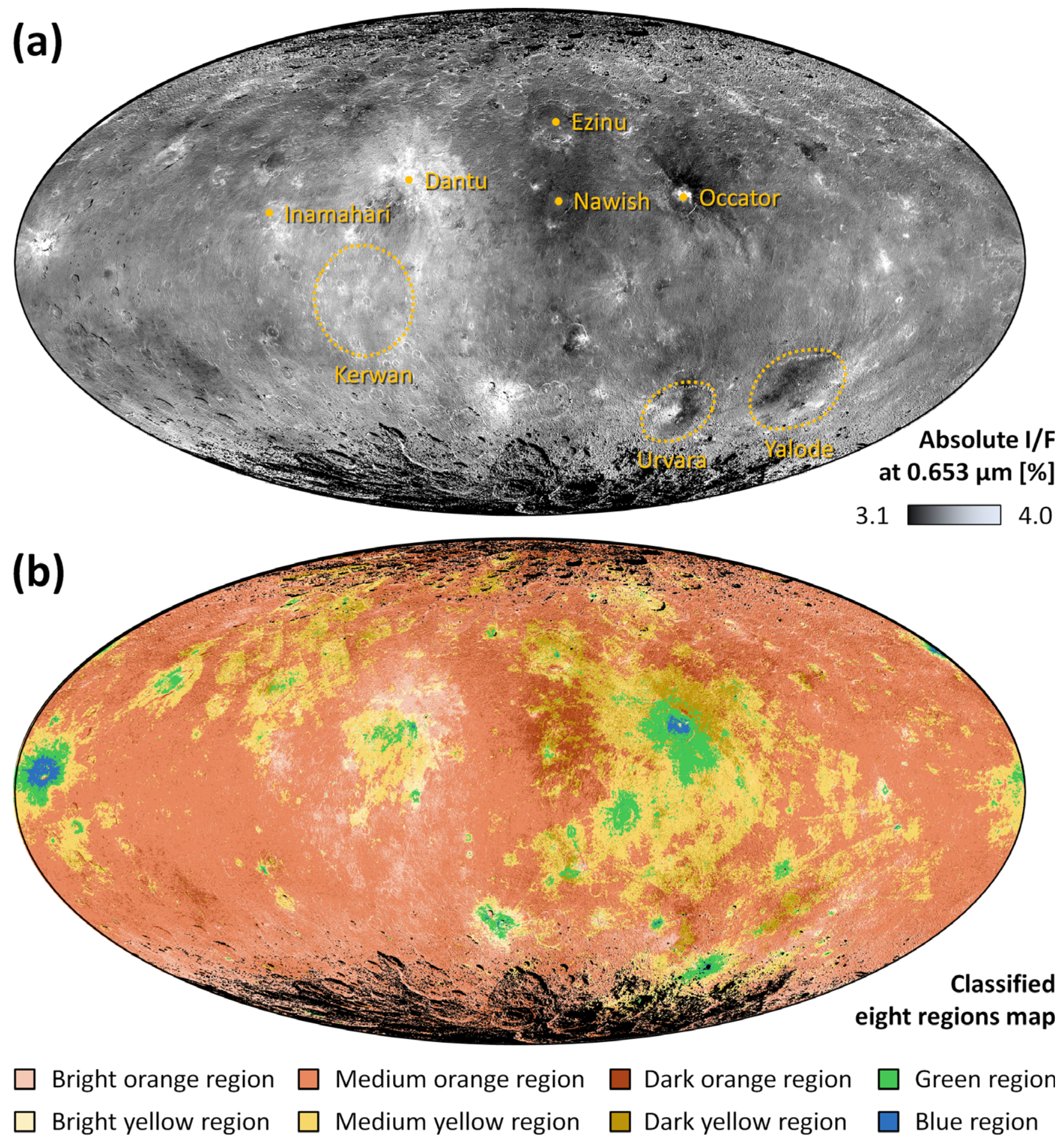

Fig. 4. Global maps of Ceres based on Dawn FC HAMO data in equal-area "Mollweide" projection. a) Absolute I/F in the $0.653 \mu \mathrm{m}$ FC filter. b) Classification map based on spectral slope and reflectance data, showing eight classes of surface units (see Table 2 for classification criteria). No spectral information about black areas near the poles is available due to high phase angle conditions or shadows.

come near the orange and yellow point clouds after shifting. From those, only A-881594 ${ }^{15}$ (CM2)

${ }^{15}$ https://www.lpi.usra.edu/meteor/metbull.php?code $=4303$ hypothetically would match the Ceres average spectra; the others show absorption bands at 0.7 and $0.9 \mu \mathrm{m}$, as well as differences in shape and position of the $2.7 \mu \mathrm{m}$ absorption relative to Ceres. 
Table 2. Reflectance criteria for subdividing orange and yellow classification areas into dark, medium, and bright classes.

\begin{tabular}{llr}
\hline Classification designation & Criteria for reflectance $R_{0.653 \mu \mathrm{m}}$ in FC filter at $0.653 \mu \mathrm{m}(\%)$ & Areal occurrence on Ceres \\
\hline Orange region (total 67.17\%) & & $9.37 \%$ \\
Dark & $R_{0.653 \mu \mathrm{m}}<3.4$ & $47.50 \%$ \\
Medium & $3.4 \leq R_{0.653 \mu \mathrm{m}}<3.8$ & $10.30 \%$ \\
Bright & $3.8 \leq R_{0.653 \mu \mathrm{m}}$ & $4.57 \%$ \\
Yellow region (total 23.04\%) & $R_{0.653 \mu \mathrm{m}}<3.4$ & $15.83 \%$ \\
Dark & $3.4 \leq R_{0.653 \mu \mathrm{m}}<3.8$ & $2.64 \%$ \\
Medium & $3.8 \leq R_{0.653 \mu \mathrm{m}}$ & \\
Bright &
\end{tabular}

Shadows, green region, and blue region (in total 9.79\%; see Table 1).

Figure 6d shows three CM1 specimens from RELAB (MET 01070, ${ }^{16}$ Moapa Valley, ${ }^{17}$ and SCO $\left.06043^{18}\right)$, which were not included in Schäfer et al. (2016) due to their smaller grain size $(<35 \mu \mathrm{m})$. MET 01070 exhibits a comparable grain size shift from $<35$ to $<75 \mu \mathrm{m}$ (green arrow) as the Murchison experiments (red arrow). Assuming a similar grain size shift, the CM1s within the dashed ellipse move toward Ceres' point cloud. In this case, a hypothetical Moapa Valley sample with a grain size of $<125 \mu \mathrm{m}$ would plot within Ceres' point cloud.

In Fig. 7, we compare all meteorite specimens found by utilizing the VIS/NIR parameter diagram (Figs. 6a, 6b, and 6d) with Ceres' VIR spectra in the $3 \mu \mathrm{m}$ region. Although comparisons of mid-IR features in VIR spectra to those of CCs measured under ambient conditions are severely limited in the $3 \mu \mathrm{m}$ region (Beck et al. 2010; Takir et al. 2013), we can show that the spectra from Takir et al. (2013) measured under vacuum and those from RELAB measured in dry air do not differ significantly in the shape and position of the $2.7 \mu \mathrm{m}$ absorption regarding the $\mathrm{CM} 1$ chondrites. The CIs are influenced by a broad $\mathrm{H}_{2} \mathrm{O}$ absorption centered around $2.9 \mu \mathrm{m}$, but the band center of the sharp OH-absorption is still unaffected compared to the Ivuna spectrum measured in vacuum and heated to $400-475 \mathrm{~K}$ by Takir et al. (2013). Therefore, comparisons of the $2.7 \mu \mathrm{m}$ absorption between the CC samples and Ceres' VIR spectra should be valid.

The two VIR spectra of Ceres plotted in Fig. 7 are taken from typical locations representing the classified surface units "yellow medium" and "orange medium," respectively (see the VIR Imaging Spectrometer Data section for locations). The band shapes of the phyllosilicate absorption are nearly equal and centered at $2.73 \mu \mathrm{m}$. This value is in agreement with the center

\footnotetext{
${ }^{16} \mathrm{https} / / /$ www.lpi.usra.edu/meteor/metbull.php?code $=16303$

${ }^{17}$ https://www.lpi.usra.edu/meteor/metbull.php?code $=48952$

${ }^{18} \mathrm{https}$ ://www.lpi.usra.edu/meteor/metbull.php?code $=46404$
}
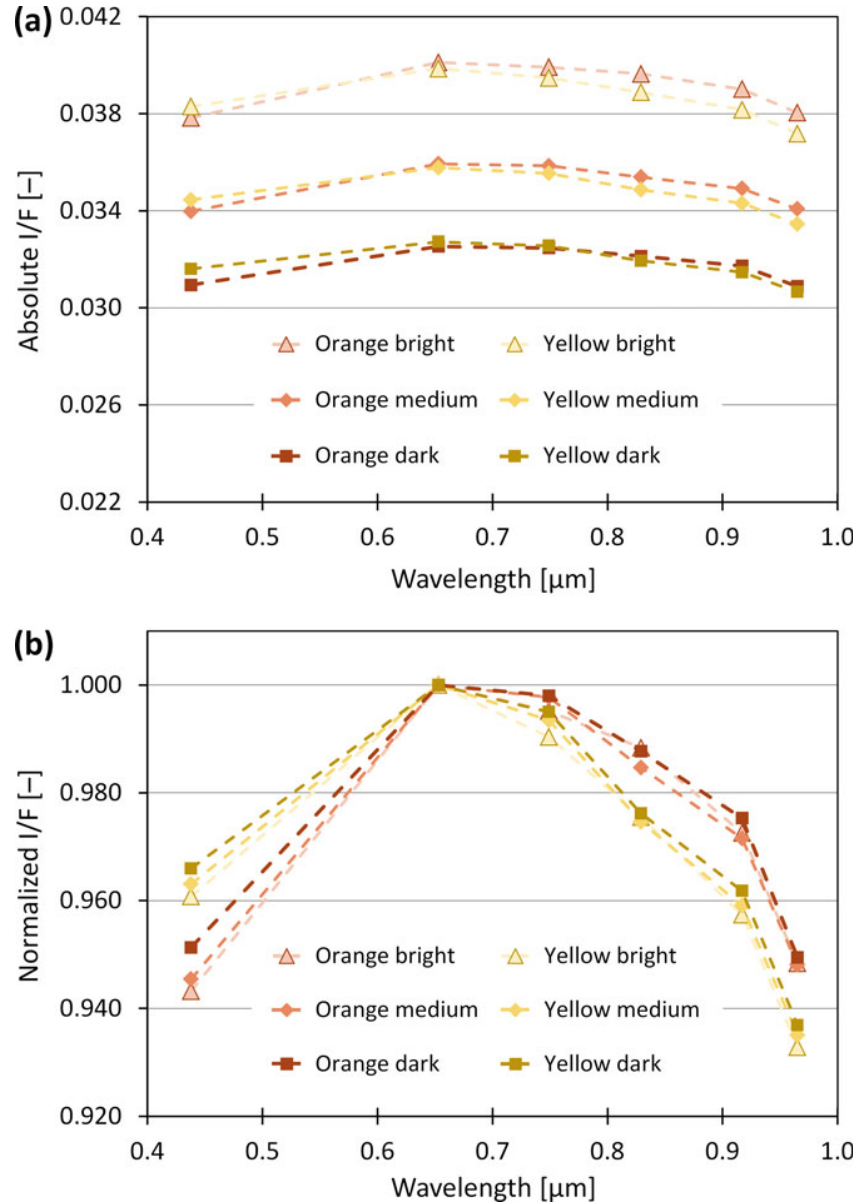

Fig. 5. Average spectra for the six classified subregions of Ceres with different absolute reflectances. a) Absolute $\mathrm{I} / \mathrm{F}$ values. b) The same spectra normalized at $0.653 \mu \mathrm{m}$.

position of $2.727 \mu \mathrm{m}$ given by Ammannito et al. (2016) who found that the position of this absorption does not vary significantly across Ceres. Also visible in the Ceres spectra is a double absorption between 3.0 and $3.1 \mu \mathrm{m}$ which was found in Dawn VIR data and interpreted as 

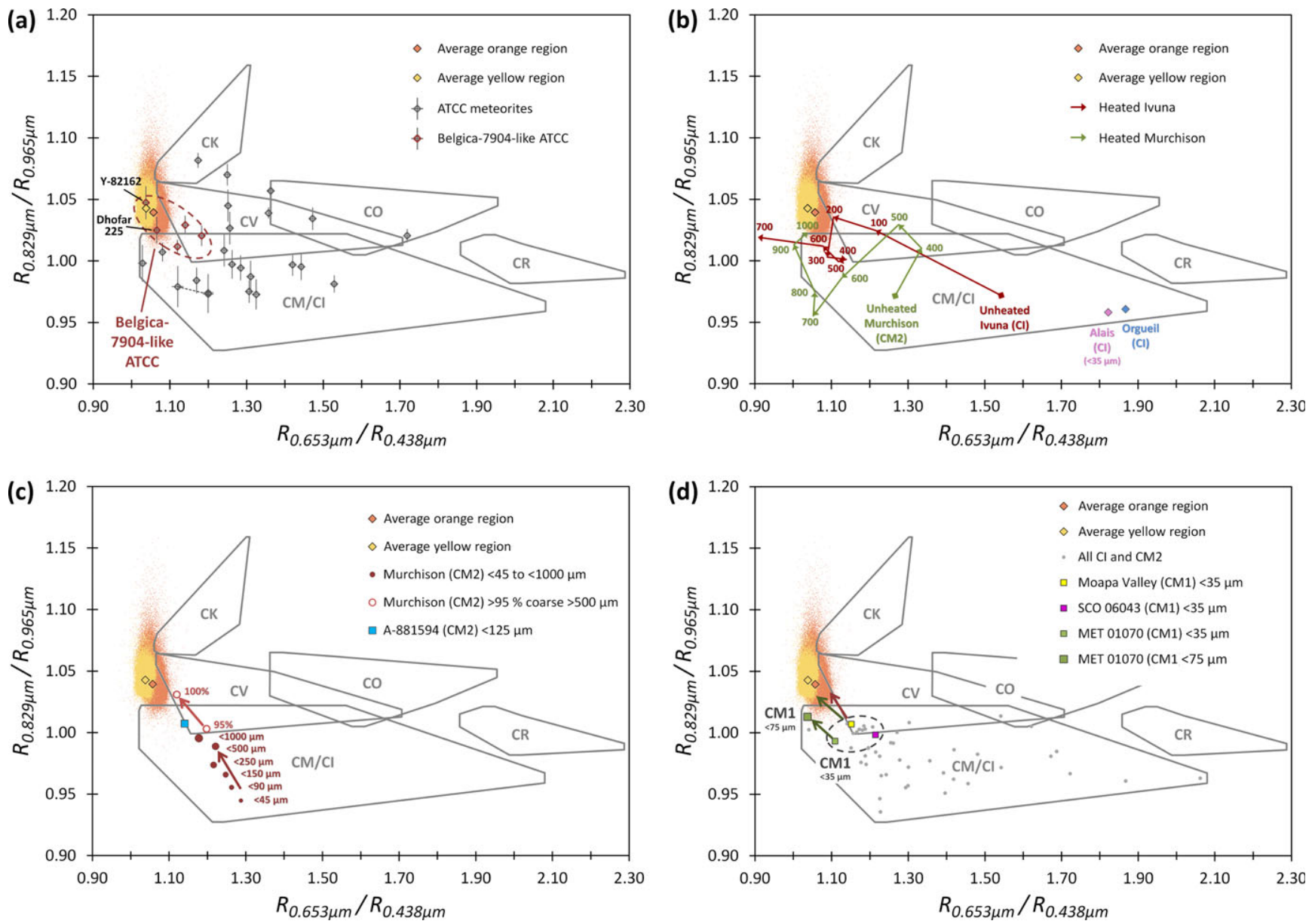

Fig. 6. Scatter plots of the two classified surface units "orange" and "yellow" in FC-based parameter space $R_{0.829 \mu \mathrm{m}} / R_{0.965 \mu \mathrm{m}}$ versus $R_{0.653 \mu \mathrm{m}} / R_{0.438 \mu \mathrm{m}}$. Polygons for different CC groups were developed in Schäfer et al. (2016). Orange and yellow diamond symbols show the position of the average spectra of the respective regions. a) Comparison with aqueously altered and thermally metamorphosed carbonaceous chondrites (ATCC), shown with error bars. Encircled by a dashed line are five Belgica-7904-like ATCC as summarized by Ivanova et al. (2010) (by oxygen isotopic criteria and their incomplete phyllosilicate transformation to olivine). b) Comparison with heating experiments of Ivuna CI (Hiroi et al. 1996) and Murchison CM chondrites (Hiroi et al. 1994). Green and red arrows show heating pathways with increasing temperatures as indicated by numbers (temperatures in degrees Celsius). Additionally, unheated CIs Orgueil and Alais are plotted for comparison. c) Range of the grain size-induced parameter shift to CCs $<125 \mu \mathrm{m}$ derived by Izawa et al. (2016) on Murchison CM2 (red arrows) and six CCs which potentially come into consideration due to their position in respect to the Ceres point clouds (see text for details). d) Position of three CM1 meteorites (squares) in relation to CM2/CI (gray dots). Two measurements of MET 01070 with different grain sizes <35 and $<75 \mu \mathrm{m}$ show a comparable grain size-induced shift (green arrow) as observed for Murchison in Fig. 6c (red arrow).

an $\mathrm{N}-\mathrm{H}$ stretching feature in $\mathrm{NH}_{4}$-bearing phyllosilicates by De Sanctis et al. (2015).

In addition, four spectra of CM1 specimens are plotted in Fig. 7 (LAP 02277, Moapa Valley, MET 01070, and SCO 06043) which show similar $2.7 \mu \mathrm{m}$ band shapes as Ceres with band center positions between 2.72 and $2.73 \mu \mathrm{m}$. Furthermore, spectra of three different CI chondrites (Ivuna, Alais, Orgueil) are plotted, which exhibit a sharp $\mathrm{OH}$-absorption centered between 2.71 and $2.72 \mu \mathrm{m}$. The three Ivuna spectra from heating experiments $\left(200,300\right.$, and $\left.600{ }^{\circ} \mathrm{C}\right)$ by Hiroi et al. (1996) also have a band center position between 2.71 and
$2.72 \mu \mathrm{m}$, but the band shape is more rounded. Moreover, two Belgica-7904-like ATCCs, Dhofar 225 and Y-82162, are plotted in Fig. 7. While plotting within Ceres' point clouds in Fig. 6a, they show a distinctly round-shaped absorption centered around $2.9 \mu \mathrm{m}$ instead of a $2.7 \mu \mathrm{m}$ band. Finally, the spectrum of CM2 Murchison experimentally heated to $1000{ }^{\circ} \mathrm{C}$ (Hiroi et al. 1994) has been added for interest, since it also plots at the edge of Ceres' point clouds in Fig. 6b. The Murchison spectrum is totally flat and lacks any absorption in the $3 \mu \mathrm{m}$ region. None of the meteorite samples in Fig. 7 shows the 3.0 $3.1 \mu \mathrm{m}$ absorption that is present in the spectra of Ceres. 


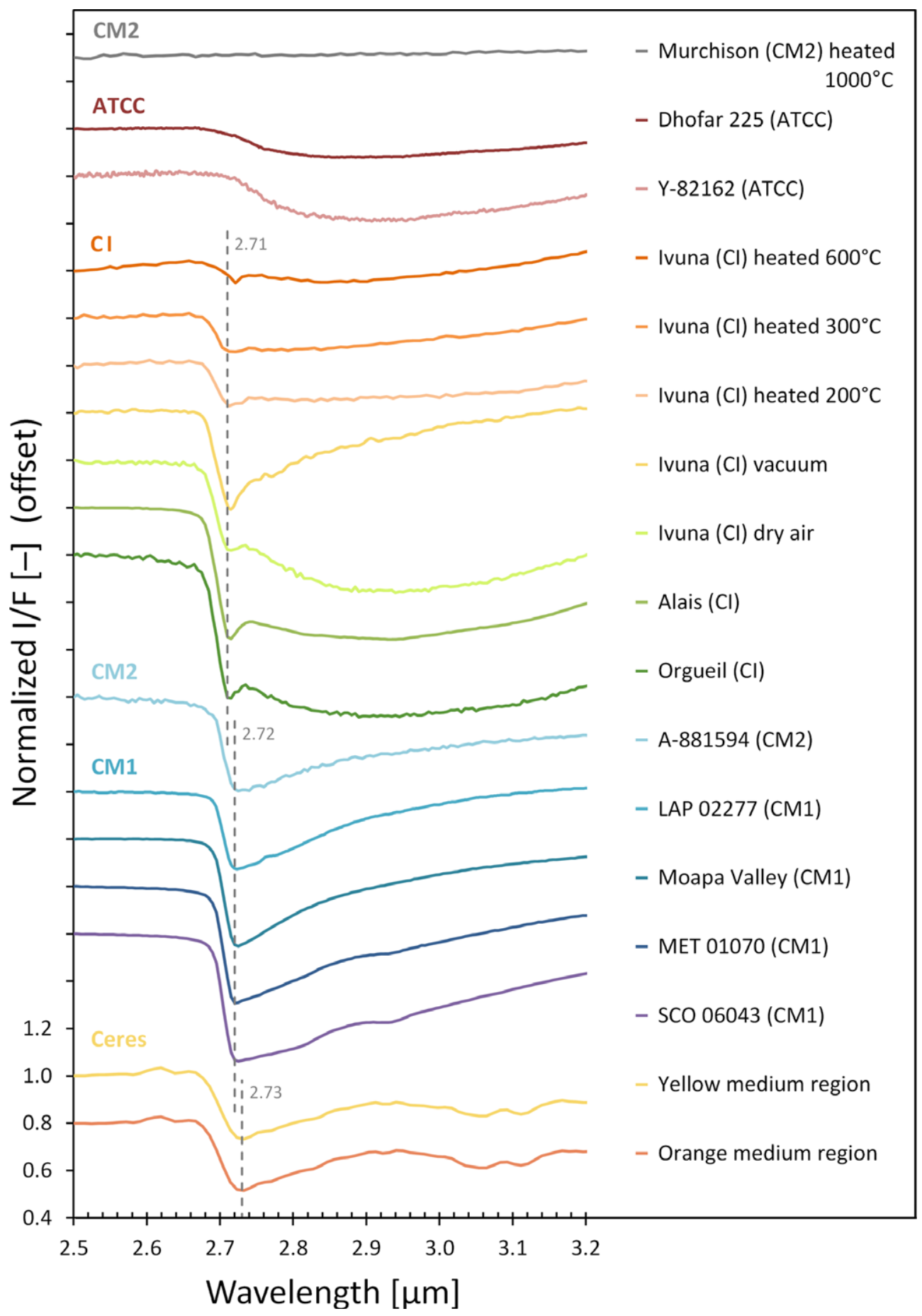

Fig. 7. Comparison of two Dawn VIR spectra of typical areas in the classified "orange medium region" and "yellow medium region" with meteorite spectra in the $2.5-3.3 \mu \mathrm{m}$ wavelength range. All spectra are normalized at $2.5 \mu \mathrm{m}$ and offset in steps of $0.2 \mathrm{I} / \mathrm{F}$ for clarity.

In Fig. 8, we compare the average absolute reflectance in the $0.653 \mu \mathrm{m}$ filter of the bright, medium, and dark classified surface units (cf. Fig. 4; Table 2) with the meteorites selected before. Figure 8 incorporates samples of different grain sizes, since for dark materials grain size does not strongly affect the reflectance in the VIS (Adams and Filice 1967), which is also shown for CM meteorites by Schäfer et al. (2016). The absolute I/F values of Y-82162, Dhofar 225, and Moapa Valley resemble best the reflectance of Ceres, 


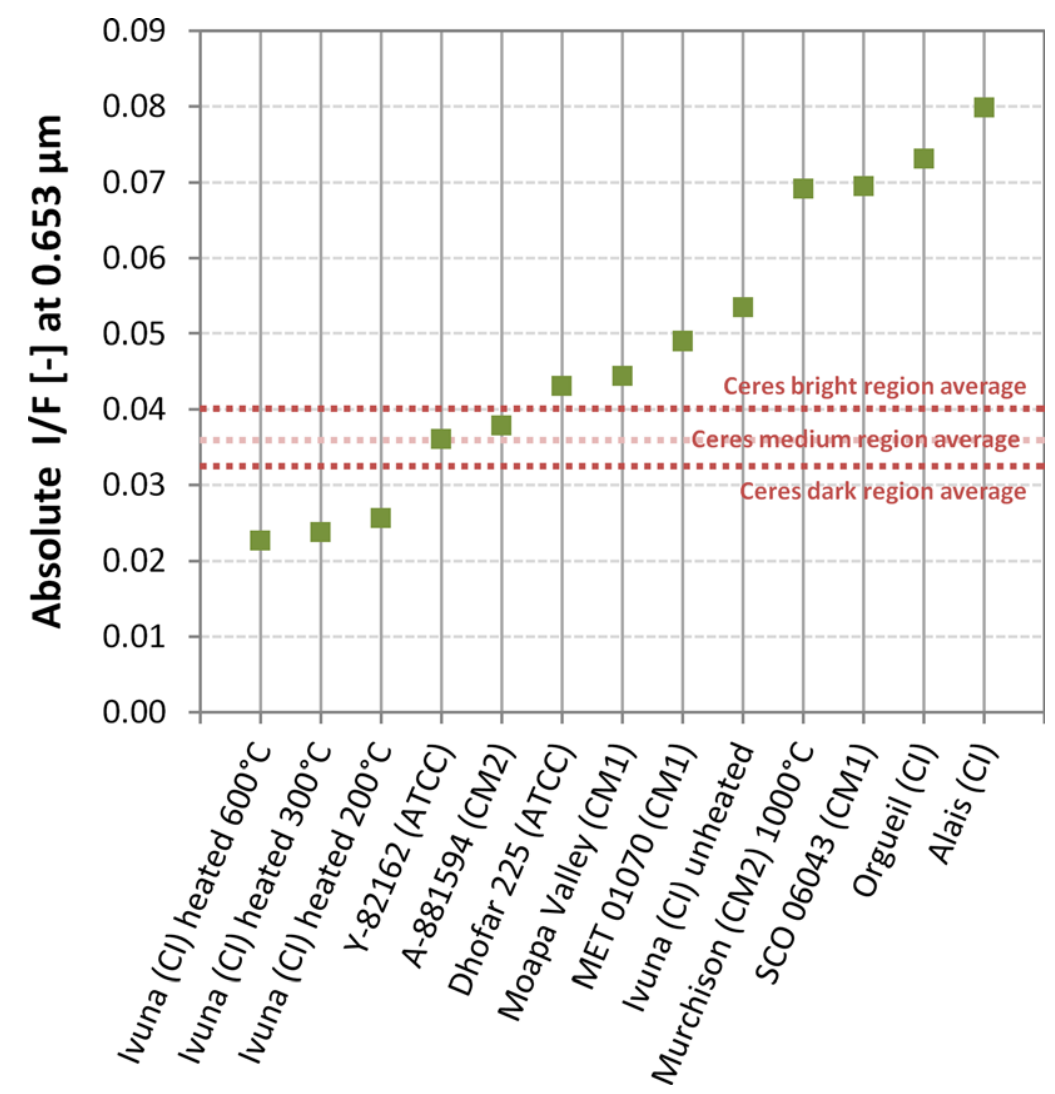

Fig. 8. Absolute reflectance in the $0.653 \mu \mathrm{m}$ filter of the selected CI, CM, and Belgica-7904-like ATCC meteorites in relation to the average $\mathrm{I} / \mathrm{F}$ of the classified dark, medium, and bright regions on Ceres (cf. Fig. 4).

followed by CM1 MET 01070. The heated Ivuna with reflectance values around 0.025 are distinctly darker than average Ceres. The CI chondrites Ivuna, Alais, and Orgueil, the CM1 SCO 06043, as well as Murchison heated to $1000{ }^{\circ} \mathrm{C}$ are considerably brighter than Ceres with reflectance values up to 0.08 .

\section{DISCUSSION}

\section{Spectral Variability of Ceres}

Our classification of the cerean surface by spectral slope $\gamma$ consists of four classes: two classes which are highly blue sloped $\left(\gamma<-10 \% \mu \mathrm{m}^{-1}\right)$ and two classes which are weakly blue sloped to red sloped $\left(\gamma \geq-10 \% \mu \mathrm{m}^{-1}\right)$ (see Fig. 2; Table 1). We applied the $\mathrm{CC}$ discrimination scheme only to regions on the cerean surface which exhibit a spectral slope $\gamma \geq-10 \% \mu \mathrm{m}^{-1}$ to exclude regions with strong negative slopes. These regions are not likely to bear pure CC-like material as can be seen by their brightness and negative spectral slope, as well as their distinctive IR spectral properties in VIR data (De Sanctis et al. 2015). Furthermore, among the complete suite of $121 \mathrm{CC}$ spectra investigated by Schäfer et al.
(2016), only three specimens exhibit such a spectral slope $\gamma<-10 \% \mu \mathrm{m}^{-1}$ (two CK4 and one CV3), which is caused by their relatively strong olivine band around $1 \mu \mathrm{m}$. Such a band is not observed in Ceres spectra, suggesting that these materials are not viable explanations for the blue spectral slope $\gamma<-10 \% \mu \mathrm{m}^{-1}$. All low petrologic grade CIs, CMs, CRs, as well as the remaining CVs and CKs show spectral slopes $\gamma>-10 \% \mu \mathrm{m}^{-1}$.

The slightly blue-sloped material in the yellow classified regions $\left(-3>\gamma \geq-10 \% \mu \mathrm{m}^{-1}\right)$ obviously is spatially connected with the blue-sloped material occurring in young craters classified as green regions. It is possibly a mixture between orange regions and green regions. The threshold between our yellow and orange region is subjectively set to visually express this connection, but does not represent a separation between pure background material and material influenced by craters. However, the absolute $\mathrm{I} / \mathrm{F}$ at $0.653 \mu \mathrm{m}$ (Fig. 4) within the yellow and orange regions does not correlate significantly with the spectral slope. This indicates that there is no simple mixing between two endmemberss crater material and background material, and that possibly some brighter component, which is spectrally neutral in the VIS/ NIR region, is admixed in some regions. 
Table 3. Modal mineralogy from X-ray diffraction of meteorites Ivuna and Y-82162 (King et al. 2015), Moapa Valley and LAP 02277 (King et al. 2017), MET 01070 and SCO 06043 (Howard et al. 2011), given in vol\%.

\begin{tabular}{|c|c|c|c|c|c|c|}
\hline Meteorite & Ivuna $(\mathrm{CI})$ & $\begin{array}{l}\text { Moapa } \\
\text { Valley (CM1) }\end{array}$ & $\begin{array}{l}\text { LAP } 02277 \\
(\mathrm{CM} 1)\end{array}$ & $\begin{array}{l}\text { MET } \\
01070 \\
(\mathrm{CM} 1)\end{array}$ & $\begin{array}{l}\text { SCO } \\
06043 \\
(\mathrm{CM} 1)\end{array}$ & $\begin{array}{l}\text { Y-82162 } \\
\text { (Belgica-7904-like) }\end{array}$ \\
\hline$\overline{\text { Fe-cronstedtite }}$ (vol\%) & Not specified & 20.4 & 20.6 & 26.3 & 21.6 & Not specified \\
\hline Mg-serpentine ${ }^{\mathrm{b}}\left(\mathrm{vol}^{\%} \%\right)$ & Not specified & 67.1 & 68.3 & 61.2 & 66.0 & Not specified \\
\hline Total phyllosilicates (vol\%) & $81-84^{\mathrm{c}}$ & 87.5 & 88.9 & 87.5 & 87.6 & $68-2=$ \\
\hline Sulfides $($ vol $\%)$ & $6-7$ & 4.4 & 2.2 & 1.2 & 2.7 & 19 \\
\hline Magnetite (vol\%) & $8-10$ & 3.1 & 1.9 & 1.8 & 1.6 & 2 \\
\hline Olivine $\left(\mathrm{vol}^{\%} \mathrm{)}\right)$ & Not detected & 4.1 & 5.3 & 7.7 & 6.3 & 11 \\
\hline Carbonates (vol\%) & $2^{\mathrm{d}}$ & 0.9 & 1.7 & 1.9 & 1.7 & Not detected ${ }^{\mathrm{e}}$ \\
\hline
\end{tabular}

\footnotetext{
${ }^{a}$ Actually Fe-rich serpentine also containing Mg, as explained in King et al. (2015) and Howard et al. (2011).

${ }^{\mathrm{b}}$ Actually Mg-rich serpentine also containing Fe, as explained in King et al. (2015) and Howard et al. (2011).

${ }^{\mathrm{c}} \mathrm{Fe}$-bearing, Mg-rich serpentine/saponite intergrowths as studied by electron beam analysis (Tomeoka 1990; Brearley 1992; Zolensky et al. 1993).

${ }^{\mathrm{d}}$ Prevailing Ca-Mg-carbonates (Johnson and Prinz 1993; Endress and Bischoff 1996).

${ }^{\mathrm{e}} 0.5 \mathrm{vol} \%$ carbonates found by optical point counting (Tomeoka et al. 1989).
}

Slope values $<-10 \% \mu \mathrm{m}^{-1}$ are associated with the youngest impact craters on Ceres, such as Oxo, Haulani, and Occator (Jaumann et al. 2016; Schmedemann et al. 2016). Schmedemann et al. (2016) found a distinct correlation between cratering model ages and spectral reflectance ratio $R_{0.438 \mu \mathrm{m}} / R_{0.965 \mu \mathrm{m}}$. This ratio strongly correlates with the spectral slope $\gamma$ used in this work and therefore also with the regions classified by us. Also from morphological aspects, the blue-sloped material is closely related to craters with the freshest appearance (Stephan et al. 2017).

\section{Grain Size Effects}

As experiments show, grain size has a significant influence on the VIS/NIR slopes of CC spectra (Johnson and Fanale 1973; Izawa et al. 2016) and thus causes a shift in the FC spectral parameters we use for CC discrimination. Therefore, hypothetically, some samples of CM- or ATCC-type chondrites below the Ceres point cloud could also move toward the Ceres region, if they contain grains distinctly larger than $125 \mu \mathrm{m}$, provided that they show a similar parameter shift as Murchison (as indicated by the red arrow in Fig. 6c). Also, if the finest grains on Ceres are depleted, additional spectral matches to Ceres are possible (Fig. 6c).

However, this seems not to be a likely scenario to us due to the highly impact gardened surface on Ceres. In fact, there is strong evidence for smaller grain sizes to be present on Ceres. Gundlach and Blum (2013) gave a grain size estimate of $120 \mu \mathrm{m}$ for disk-integrated Ceres deduced by thermal inertia from telescopic observations. Recent observations by the VIR instrument indicate grain sizes varying between $<60$ and $<120 \mu \mathrm{m}$ for the average terrain based on thermal modeling (Raponi, personal communication). Also, the low regolith vapor diffusivity inferred by GRaND supports small grain sizes of $<100 \mu \mathrm{m}$ (Prettyman et al. 2017).

Furthermore, regions covered with coarse only material, e.g., boulders or outcrops, should be spatially underrepresented on Ceres and may be covered in finegrained dust. As we use spatially averaged spectra of the entire yellow and orange regions, these should be statistically insignificant. For all these reasons, our CC discrimination diagram should be applicable, since it is based on laboratory spectra of powders ranging from $<75$ to $<125 \mu \mathrm{m}$ (including all the fines).

\section{Comparison to Meteorites and Origins of Observed Features}

\section{Belgica-7904-Like Meteorites}

Although the Belgica-7904-like meteorites Dhofar 225 and Y-82162 match best Ceres background material by their position in the $\mathrm{CC}$ discrimination diagram (Fig. 6a) and by their reflectance values (Fig. 8), they show significant differences concerning the phyllosilicates since they lack the sharp $2.7 \mu \mathrm{m}$ absorption of Ceres (Fig. 7). Instead, they exhibit an absorption feature at $0.7 \mu \mathrm{m}$ indicating Fe-bearing phyllosilicates (e.g., Cloutis et al. 2012; Schäfer et al. 2016), which is not present in Dawn FC data of Ceres. For these spectral reasons, the Belgica-7904-like meteorites can be excluded as an analogue material for Ceres.

The position of the Belgica-7904-like group between the highly thermally metamorphosed olivine-bearing CKs and the aqueously altered olivine-poor CMs (Fig. 6a) can be explained by their modal mineralogy. For example, Y-82162 contains $11 \mathrm{vol} \%$ of olivine (Table 3) (King et al. 2015), which recrystallized at the expense of 
phyllosilicates resulting in structurally disordered, dehydrated, and dehydroxylated phyllosilicates (Akai 1990; Ivanova et al. 2010; King et al. 2015). For this reason, the absorptions of olivine at 1.05 and $1.25 \mu \mathrm{m}$ and those of phyllosilicates at 0.7 and $0.9 \mu \mathrm{m}$ coalesce to a broad absorption extending from $\sim 0.8$ to $\sim 1.3 \mu \mathrm{m}$ (Cloutis et al. 2012), which is expressed in the parameter $R_{0.829 \mu \mathrm{m}} / R_{0.965 \mu \mathrm{m}}$ (Schäfer et al. 2016).

Whether an olivine-phyllosilicate mixture, as indicated by the spectral match of Belgica-7904-like meteorites in the VIS/NIR region, is an analogue for Ceres background, is a subject which needs discussion in the context of partial serpentinization of an olivinebearing protolith (e.g., supplementary ordinary chondrite material) and in relationship to the $\mathrm{Ca}-\mathrm{Mg}$-carbonate findings in Ceres average spectrum (De Sanctis et al. 2015) being possibly produced by this process. But exploring this is beyond the scope of this paper.

\section{$\mathrm{Cl}$ Meteorites}

The unheated CI specimens Alais, Orgueil, and Ivuna show considerably higher values for the $R_{0.829 \mu \mathrm{m}} /$ $R_{0.965 \mu \mathrm{m}}$ parameter (Fig. 6b) than Ceres and have a much higher reflectance in the VIS (Fig. 8). Also the shape and position of their $2.7 \mu \mathrm{m}$ absorption feature (between 2.71 and $2.72 \mu \mathrm{m}$ ) does not perfectly match Ceres VIR spectra (Fig. 7). This spectrally disqualifies the available CIs as an analogue for Ceres' background material.

However, the Ivuna samples heated to 200, 300, and $600{ }^{\circ} \mathrm{C}$ plot nearest the Ceres position in our discrimination diagram (Fig. 6b). Also the shape of the $2.7 \mu \mathrm{m}$ absorption of the samples heated to 200 and $300{ }^{\circ} \mathrm{C}$ resembles Ceres spectra (Fig. 7), but the band center remains unchanged in respect to the unheated Ivuna and does not entirely fit Ceres either. The Ivuna sample heated to $600{ }^{\circ} \mathrm{C}$ does not match shape and position of Ceres' $2.7 \mu \mathrm{m}$ band at all. Furthermore, the VIS reflectance values for all heated Ivuna samples are considerably lower than Ceres background material (Fig. 8). All in all, the heated Ivuna samples do not exhibit good matches to spectra of Ceres.

The CI Ivuna consists predominantly of phyllosilicates $(>80 \%)$ and minor magnetite, sulfides, and carbonates (see Table 3) (King et al. 2015). The phyllosilicates are specified as Fe-bearing, Mg-rich serpentine/saponite intergrowths (Tomeoka 1990; Brearley 1992; Zolensky et al. 1993). This is consistent with the reflectance spectra of CIs indicating $\mathrm{Mg}$-rich rather than Fe-rich phyllosilicates, as they show the sharp $\mathrm{Mg}-\mathrm{OH}$ serpentine absorption at $2.71 \mu \mathrm{m}$ (Beck et al. 2010; Takir et al. 2013) and lack absorptions at $0.7 \mu \mathrm{m}\left(\mathrm{Fe}^{2+}-\mathrm{Fe}^{3+}\right.$ metal-metal charge transfers $)$ and $0.9 \mu \mathrm{m}\left(\mathrm{Fe}^{2+}\right.$ ligand field transitions).
Takir et al. (2013) found that the $2.7 \mu \mathrm{m}$ absorption in the $\mathrm{CM} / \mathrm{CI}$ meteorites is characteristic of their phyllosilicate mineralogy and hence related to the aqueous alteration sequence of $\mathrm{CMs} / \mathrm{CIs}$ (McSween et al. 2017), with the Mg-endmembers predominant in highly aqueous altered Ivuna being positioned at $2.71 \mu \mathrm{m}$ and the Fe-endmembers in the least altered CMs being positioned at $2.80 \mu \mathrm{m}$. But this is a general trend and does not allow us to directly determine the $\mathrm{Mg}-/ \mathrm{Fe}-$ content as the texturally and structurally complex phyllosilicate intergrowths in $\mathrm{CI} / \mathrm{CM}$ chondrite matrix (Müller et al. 1979; Tomeoka and Buseck 1988) are not directly comparable to known terrestrial analogues (Howard et al. 2011). Therefore, Ceres' higher band center position $(2.72-2.73 \mu \mathrm{m})$ indicates a slightly different phyllosilicate mineralogy than the CIs $(2.71-2.72 \mu \mathrm{m})$, which is more consistent with the less altered CM1s (cf. next section).

Spectral comparisons between Ceres' and Ivuna's carbonates are very limited since the carbonate absorption between 3.4 and $3.5 \mu \mathrm{m}$ in the Ivuna spectrum is influenced by organics and the absorption near $3.9 \mu \mathrm{m}$ is too weak to be firmly evaluated (De Sanctis, personal communication). The weak carbonate absorptions can be explained by the low total carbonate content $(\sim 2 \%)$ of Ivuna. Nevertheless, the carbonates prevailing in Ivuna (Johnson and Prinz 1993; Endress and Bischoff 1996) are mineralogically similar to the $\mathrm{Ca}-\mathrm{Mg}$-carbonate (magnesite and dolomite) commonly seen on Ceres by VIR (De Sanctis et al. 2016).

\section{CM1 Meteorites}

All available VIS/NIR spectra of CM1-type meteorites (Moapa Valley, MET 01070, and SCO 06043) plot in the upper left edge of the CM/CI field of our CC discrimination diagram, albeit outside the point clouds of Ceres' orange and yellow surface material (Fig. 6d). However, these spectra are measured using relatively fine grain sizes $(<75$ and $<35 \mu \mathrm{m})$. When assuming a shift toward the upper left, as shown for MET 01070 (green arrow) and Murchison (red arrow in Fig. 6c), the CM1s with grain sizes larger than $<35 \mu \mathrm{m}$ would move toward Ceres' position in the diagram, especially Moapa Valley, which would plot within the point cloud of Ceres when assuming a comparable grain size of $<120 \mu \mathrm{m}$ (see the Grain Size Effects section).

Strong evidence for the CM1s being similar to Ceres surface materials is indicated by their mid-IR spectra, which exhibit a strong and similarly shaped $2.7 \mu \mathrm{m}$ absorption to Ceres, also centered between 2.72 and $2.73 \mu \mathrm{m}$ (Fig. 7). Additionally, regarding the absolute reflectance at $0.653 \mu \mathrm{m}$, Moapa Valley is close to Ceres average reflectance, whereas MET 01070 is slightly, and SCO 06043 is distinctly brighter, than Ceres (Fig. 8). 
Moreover, MET 01070, SCO 06043, and LAP 02277 show the Fe-phyllosilicate absorption at $0.7 \mu \mathrm{m}$ (Takir et al. 2013; McAdam et al. 2015) not visible in Ceres' spectra. Thus, the remaining CM1 Moapa Valley is a probable candidate for material occurring on Ceres.

The CM1 chondrites represent highly aqueous altered CM2 material and are the most altered endmember of the CM group as described by Zolensky et al. (1997) and Rubin et al. (2007). They contain nearly $90 \%$ phyllosilicates and minor olivine, sulfide, magnetite, and carbonate (cf. Table 3) (King et al. 2017). CM1s are the most phyllosilicate-rich material among the meteorite collection, containing some relict olivine $(<8 \%)$. Moapa Valley, best matching Ceres spectrally, contains only $4.1 \%$ olivine. But this olivine content seems too low to be spectrally expressed, as there is no olivine absorption visible in the CM1 spectra from RELAB and also not in the CM1 spectra from Takir (cf. fig. 5 in Takir et al. 2013). The phyllosilicates are composed of $\sim 2 / 3$ of the $\mathrm{Mg}$-rich variety, which is consistent with their progressive aqueous alteration, and also being consistent with the position of the phyllosilicate absorption between 2.72 and $2.73 \mu \mathrm{m}$ as shown for LAP 02277 in Takir et al. (2013).

The carbonate content of the CM1s $(<2 \%)$ is too low to be spectrally detectable, as we found no absorption near $3.9 \mu \mathrm{m}$ in any of the CM1 spectra. A qualitative specification of the carbonates in the CM1 is only given for EET 83334 (Zolensky et al. 1997), a specimen not included in our study due to the lack of reflectance spectra for it. For this specimen, calcite and dolomite in equal proportions, along with more complex Ca-Mg-Fe-Mn-Ni-carbonates are described. Also Rubin et al. (2007) states a general trend from pure Ca-carbonates in the least altered $\mathrm{CM} 2 \mathrm{~s}$ to more complex Ca-Mg-Fe-Mn-bearing carbonates in the highly aqueous altered $\mathrm{CM}$ chondrites. These $\mathrm{Ca}-\mathrm{Mg}$ carbonates are consistent with the carbonate mineralogy detected by De Sanctis et al. (2015) in the average spectrum of Ceres.

\section{CM2 A-881594}

At last, we want to remark that we found one CM specimen, A-881594, which matches the spectral properties of Ceres for the $2.7 \mu \mathrm{m}$ absorption and the absolute reflectance at $0.653 \mu \mathrm{m}$ (Fig. 7), but needs the assumption of grain sizes on Ceres of at least $<500 \mu \mathrm{m}$ (Fig. 6c). Yet, this is not consistent with the findings that Ceres' surface is dominated by grain sizes $<125 \mu \mathrm{m}$ (see the Grain Size Effects section). Another hypothetical solution could be that the reported grain size of $<125 \mu \mathrm{m}$ for A-881594 is just given as an upper limit, and that the sample became finer-grained during grinding. Then the position of A-881594 in our discrimination diagram could be similarly shifted as seen for MET 01070 (Fig. 6d), resulting in an overlap with Ceres' point clouds.

Aside from these speculations on grain sizes, it is notable that A-881594 exhibits the same phyllosilicate absorption between 2.72 and $2.73 \mu \mathrm{m}$ as the CM1 (Fig. 7) and in the VIS parameters it plots on nearly the same position as the CM1 Moapa Valley (Figs. 6c and $6 \mathrm{~d}$ ), which has a grain size of $<35 \mu \mathrm{m}$. The position and shape of the $2.7 \mu \mathrm{m}$ absorption indicates a Mg-rich phyllosilicate mineralogy as in the CM1s due to a high degree of aqueous alteration. To our knowledge, there is neither a mineralogical nor petrological description of A-881594, which could better elucidate the extent of any relationship to CM1 chondrites.

\section{Implications for Ceres and the Origin of $\mathbf{C M}$}

The spectral signature of Ceres' background material indicates CM1 material rather than CI material as the closest available analogue. The link between CM1 and Ceres raises some important aspects for Ceres, as well as for the parent body alteration of CM material.

The alteration sequence from the least altered CM2 to highly altered CM1 or CI material is characterized by a change in phyllosilicate mineralogy, transforming from Fe-rich phyllosilicates to Mg-rich phyllosilicates, the $\mathrm{Fe}$ being incorporated into Fe-oxides, as summarized by McSween et al. (2017), and verified by many researchers over the years (e.g., McSween 1979; Tomeoka and Buseck 1985; Howard et al. 2009, 2011). The CM1 consists of nearly $90 \%$ phyllosilicates, twothirds of them Mg-rich (Table 3). They evidently evolved by the aqueous alteration of CM2, as indicated by their petrological texture, showing relict chondrules completely altered to phyllosilicates (Zolensky et al. 1997; Rubin et al. 2007), and by their oxygen isotope ratios plotting within the CM group (Irving et al. 2009). Although the CIs have a similar Mg-rich phyllosilicate mineralogy and carbonate content as the CM1s (Table 3), they exhibit a completely different oxygen isotope signature (Clayton and Mayeda 1999) and show no sign that chondrules ever existed. This indicates that they formed by different processes, likely on different parent bodies. Unfortunately, we have no compositional explanation for the spectral differences between the pristine CIs and the CM1s in the VIS (Figs. 6b, 6d, and 8), especially not for the significant difference in the drop-off shortward of $0.6 \mu \mathrm{m}$. Somewhat speculatively, the short-wavelength spectral differences may be controlled mainly by differences in the nature of the organic materials present. Organic materials containing a mixture of $\mathrm{sp}^{2}$ - and $\mathrm{sp}^{3}$-bonded carbon produce very strong absorptions at ultraviolet wavelengths, the long- 
wavelength "wings" of which extend well into the visible region (e.g., Applin et al. 2018).

Speaking against the CIs, aside from not perfectly matching Ceres' $2.7 \mu \mathrm{m}$ absorption, is, that they need heating between 200 and $300{ }^{\circ} \mathrm{C}$ to spectrally match Ceres' background material in the VIS/NIR region. The scenarios explored by geochemical modeling (CastilloRogez et al. 2018) cover temperatures of up to $120{ }^{\circ} \mathrm{C}$, but a temperature of $15{ }^{\circ} \mathrm{C}$ is favored since Neveu et al. (2017) showed that exchange of ammonia with cations in clays happens preferentially at temperatures lower than $50^{\circ} \mathrm{C}$. Furthermore, it is difficult to expect temperatures in excess of $100{ }^{\circ} \mathrm{C}$ in an open hydrothermal medium like Ceres (Castillo-Rogez, personal communication). Thus, the remaining possibility for heating would be impacts. Little is known about the temperatures affecting the regolith by impacts on ice-bearing bodies, but Bowling et al. (2016) determined via simulations that maximum temperatures occurring in Occator did not exceed $\sim 70{ }^{\circ} \mathrm{C}$.

Ceres' present surface mineralogy can be explained by both $\mathrm{CM}$ and $\mathrm{CI}$ compositions as possible precursor material, as shown by McSween et al. (2017) and explored by Castillo-Rogez et al. (2018) for CIs and Neveu et al. (2017) for CM2s. In these scenarios, adding a $\mathrm{NH}_{3}$ and $\mathrm{CO}_{2}$ source explains the occurrence of ammoniated clays on Ceres (De Sanctis et al. 2015) and the formation of $\mathrm{Na}_{2} \mathrm{CO}_{3}$ in Occator detected by VIR (De Sanctis et al. 2016) as a late precipitate from aqueous solutions.

Ceres' carbonate mineralogy can also result from a CM1 protolith (CM2). Inspections of MET 01070 (Rubin et al. 2007) and EET 83334 (Zolensky et al. 1997) show that these CM1 specimens are depleted in $\mathrm{Ca}, \mathrm{Na}$, and $\mathrm{K}$ relative to $\mathrm{CM} 2$. These elements likely were removed by leaching the CM2 and enriched in hydrothermal fluids circulating in Ceres' interior, being consistent with fluid compositions modeled by CastilloRogez et al. (2018). The leached $\mathrm{Ca}$ is possibly incorporated in the Ca-Mg-carbonates commonly seen on Ceres' surface (De Sanctis et al. 2015, 2016) and Na in the Na-carbonates occurring in Occator (De Sanctis et al. 2016), which could have formed by a sudden impact-induced pressure release. This supports that Ceres' upper layer is enriched in carbonates relative to $\mathrm{CM} 1$, as, contrary to Ceres, the CM1 spectra do not show any sign of carbonate absorption features. The processes described above for parent body alteration from CM2 to CM1 are discussed in detail by Zolensky et al. (1997), who suggest the interesting idea of an "asteroidal caliche" in this context.

Another hint that CM1s originate from a large body like Ceres is their special petrologic texture. The relict chondrules in $\mathrm{CM} 1 \mathrm{~s}$ are flattened and show orientation; moreover, the meteorites exhibit a shaly appearance (Zolensky et al. 1997; Rubin et al. 2007; Irving et al. 2009). The alignment of particles increases with higher degree of aqueous alteration in the CM group (Rubin 2012). Whether this alignment was generated by compaction or by impacts is currently unresolved (Zolensky et al. 1997; Rubin 2012); however, Zolensky et al. (1997) concluded that this texture is rather a result of compaction. On Ceres, possibly both scenarios coexist, since the size of the protoplanet allows both compaction due to burial pressure in the deeper interior, as well as a wide variety of impact structures of all sizes on the surface. Both processes can be connected to advanced aqueous alteration (Rubin 2012).

\section{SUMMARY AND CONCLUSIONS}

1. Observations of multispectral data from the Dawn FC in the $0.4-1.0 \mu \mathrm{m}$ wavelength region show that more than $90 \%$ of the observable cerean surface is covered by material exhibiting weakly negative to positive spectral slopes $\left(\gamma \geq-10 \% \mu \mathrm{m}^{-1}\right)$, denoted as Ceres' background material.

2. The 0.7 and $0.9 \mu \mathrm{m}$ absorption bands indicative of Fe-bearing phyllosilicates were not found in FC color mosaics of Ceres.

3. Regarding the most highly aqueous altered meteorites, we found that Ceres' background material has more spectral affinity to CM1 meteorites than to CIs, with the best match to the CM1 specimen Moapa Valley.

4. Moapa Valley and other CM1s show a petrologic texture (alignment of relict chondrules and shaly appearance), which suggests their formation on a larger body like Ceres.

5. All this indicates that an alteration of $\mathrm{CM} 2$ material to CM1 on Ceres is likely.

6. Compositional differences between Ceres and CCs, such as the lack of $\mathrm{NH}_{4}$-clays in meteorites, enrichment in Ca-Mg-carbonates at the overall surface, and the late precipitating $\mathrm{Na}$-carbonate at Occator (De Sanctis et al. 2015, 2016), are consistent with geochemical models starting from $\mathrm{CM} 2$ or CI material, interacting with an aqueous solution containing $\mathrm{NH}_{3}$ and $\mathrm{CO}_{2}$ (Neveu et al. 2017; Castillo-Rogez et al. 2018).

Acknowledgments - The authors thank the three referees Bethany Ehlmann, Lucy A. McFadden, and Paul G. Lucey for their valuable comments that helped to improve the manuscript. M. S. and T. S. thank Julie Castillo-Rogez for helpful comments on Ceres' thermal evolution, Cristina De Sanctis for helpful comments on 
carbonates, and Kurt Mengel for helpful discussions and comments on meteorites. The authors thank Driss Takir for providing his vacuum spectra of meteorites. The authors thank the Dawn Flight team and the Dawn FC and VIR instrument teams at MPS, DLR, and INAF for successful operations at Ceres. The Dawn FC project is financially supported by Max Planck Society, German Aerospace Center (DLR), and NASA/JPL. M. S. is grateful to Ralf Jaumann, DLR, for enabling this work. T. S. thanks the International Max Planck Research School (IMPRS) at MPS for funding. E. A. C. thanks the Canadian Space Agency, the Canada Foundation for Innovation, the Manitoba Research Innovation Fund, the Natural Sciences and Engineering Research Council of Canada, and the University of Winnipeg for supporting this study. We are thankful to all the numerous authors who contributed their spectra to RELAB facility and those people who kept running these facilities and enabled access to the data. T. S. and M. S. thank A. S. for nighttime peace.

\section{Editorial Handling-Dr. Thomas McCord}

\section{REFERENCES}

Adams J. B. and Filice A. L. 1967. Spectral reflectance 0.4 to 2.0 microns of silicate rock powders. Journal of Geophysical Research 72:5705-5715.

Akai J. 1990. Mineralogical evidence of heating events in Antarctic carbonaceous chondrites, Y-86720 AND Y82162. Proceedings of the NIPR Symposium on Antarctic Meteorites 3:55-68.

Ammannito E., DeSanctis M. C., Ciarniello M., Frigeri A., Carrozzo F. G., Combe J.-P., Ehlmann B. L., Marchi S., McSween H. Y., Raponi A., Toplis M. J., Tosi F., CastilloRogez J. C., Capaccioni F., Capria M. T., Fonte S., Giardino M., Jaumann R., Longobardo A., Joy S. P., Magni G., McCord T. B., McFadden L. A., Palomba E., Pieters C. M., Polanskey C. A., Rayman M. D., Raymond C. A., Schenk P. M., Zambon F., and Russell C. T. 2016. Distribution of phyllosilicates on the surface of Ceres. Science 353:aaf4279. https://doi.org/10.1126/science.aaf4279.

Applin D. M., Cloutis E. A., and Izawa M. R. M. 2016. Reststrahlen bands near 3 microns in car-bon-bearing compounds and applications to asteroid spectroscopy (abstract \#2557). 47th Lunar and Planetary Science Conference. CD-ROM.

Applin D. M., Izawa M. R. M., Cloutis E. A., Gillis-Davis J., Pitman K. M., Roush T. L., Hendrix A. R., and Lucey P. G. 2018. Ultraviolet spectral reflectance of carbonaceous materials. Icarus 307:40-82. https://doi.org/10.1016/j.ica rus.2018.02.012.

Beck P., Quirico E., Montes-Hernandez G., Bonal L., Bollard J., Orthous-Daunay F.-R., Howard K. T., Schmitt B., Brissaud O., Deschamps F., Wunder B., and Guillot S. 2010. Hydrous mineralogy of CM and CI chondrites from infrared spectroscopy and their relationship with low albedo asteroids. Geochimica et Cosmochimica Acta 74:4881-4892. https://doi.org/10.1016/j.gca.2010.05.020.
Bowling T. J., Ciesla F. J., Marchi S., Johnson B. C., Davidson T. M., Castillo-Rogez J. C., De Sanctis M. C., Raymond C. A., and Russell C. T. 2016. Impact induced heating of Occator crater on asteroid 1 Ceres (abstract \#2268). 47th Lunar and Planetary Science Conference. CD-ROM.

Brearley A. J. 1992. Mineralogy of fine-grained matrix in the Ivuna carbonaceous chondrite (abstract). 23rd Lunar and Planetary Science Conference. p. 153.

Bus S. and Binzel R. 2002a. Phase II of the small main-belt asteroid spectroscopic survey: A feature-based taxonomy. Icarus 158:146-177. https://doi.org/10.1006/icar.2002.6856.

Bus S. and Binzel R. 2002b. Phase II of the small main-belt asteroid spectroscopic survey: The observations. Icarus 158:106-145. https://doi.org/10.1006/icar.2002.6857.

Calvin W. M. and King T. V. V. 1997. Spectral characteristics of iron bearing phyllosilicates: Comparison to Orgueil (CI1), Murchison and Murray (CM2). Meteoritics \& Planetary Science 32:693-701.

Castillo-Rogez J. C., Neveu M., McSween H., Fu R., Toplis M., and Prettyman T. 2018. Insights into Ceres' evolution from surface composition. Meteoritics \& Planetary Science. forthcoming.

Chapman C. R. and Salisbury J. W. 1973. Comparison of meteorite and asteroid spectral reflectivities. Icarus 19:507522. https://doi.org/10.1016/0019-1035(73)90078-X.

Clayton R. N. and Mayeda T. K. 1999. Oxygen isotope studies of carbonaceous chondrites. Geochimica et Cosmochimica Acta 63:2089-2104. https://doi.org/10.1016/ S0016-7037(99)00090-3.

Cloutis E. A., Hudon P., Hiroi T., and Gaffey M. J. 2012. Spectral reflectance properties of carbonaceous chondrites 4: Aqueously altered and thermally metamorphosed meteorites. Icarus 220:586-617. https://doi.org/10.1016/j.ica rus.2012.05.018.

De Sanctis M. C., Coradini A., Ammannito E., Filacchione G., Capria M. T., Fonte S., Magni G., Barbis A., Bini A., Dami M., Ficai-Veltroni I., and Preti G. 2011. The VIR spectrometer. In The Dawn mission to minor planets 4 Vesta and 1 Ceres, edited by Russell C. and Raymond C. New York: Springer. pp. 329-369.

De Sanctis M. C., Ammannito E., Raponi A., Marchi S., McCord T. B., McSween H. Y., Capaccioni F., Capria M. T., Carrozzo F. G., Ciarniello M., Longobardo A., Tosi F., Fonte S., Formisano M., Frigeri A., Giardino M., Magni G., Palomba E., Turrini D., Zambon F., Combe J.P., Feldman W., Jaumann R., McFadden L. A., Pieters C. M., Prettyman T., Toplis M., Raymond C. A., and Russell C. T. 2015. Ammoniated phyllosilicates with a likely outer solar system origin on (1) Ceres. Nature 528:241-244. https://doi.org/10.1038/nature16172.

De Sanctis M. C., Raponi A., Ammannito E., Ciarniello M., Toplis M. J., McSween H. Y., Castillo-Rogez J. C., Ehlmann B. L., Carrozzo F. G., Marchi S., Tosi F., Zambon F., Capaccioni F., Capria M. T., Fonte S., Formisano M., Frigeri A., Giardino M., Longobardo A., Magni G., Palomba E., McFadden L. A., Pieters C. M., Jaumann R., Schenk P., Mugnuolo R., Raymond C. A., and Russell C. T. 2016. Bright carbonate deposits as evidence of aqueous alteration on (1) Ceres. Nature 536:54-57. https://doi.org/10.1038/nature18290.

De Sanctis M. C., Ammannito E., McSween H. Y., Raponi A., Marchi S., Capaccioni F., Capria M. T., Carrozzo F. G., Ciarniello M., Fonte S., Formisano M., Frigeri A., 
Giardino M., Longobardo A., Magni G., McFadden L. A., Palomba E., Pieters C. M., Tosi F., Zambon F., Raymond C. A., and Russell C. T. 2017. Localized aliphatic organic material on the surface of Ceres. Science 335:719-722. https://doi.org/10.1126/science.aaj2305.

DeMeo F. E., Binzel R. P., Slivan S. M., and Bus S. J. 2009. An extension of the Bus asteroid taxonomy into the nearinfrared. Icarus 202:160-180. https://doi.org/10.1016/j.ica rus. 2009.02.005.

Endress M. and Bischoff A. 1996. Carbonates in CI chondrites: Clues to parent body evolution. Geochimica et Cosmochimica Acta 60:489-507. https://doi.org/10.1016/ 0016-7037(95)00399-1.

Fornasier S., Lazzarin M., Barbieri C., and Barucci M. A. 1999. Spectroscopic comparison of aqueous altered asteroids with CM2 carbonaceous chondrite meteorites. Astronomy \& Astrophysics Supplementary Series 135:65-73. https://doi.org/10.1051/aas:1999161.

Gaffey M. J. and McCord T. B. 1977. Asteroid surface materials: Mineralogical characterizations and cosmological implications. Proceedings, 8th Lunar Science Conference. pp. 113-143.

Gundlach B. and Blum J. 2013. A new method to determine the grain size of planetary regolith. Icarus 223:479-492. https://doi.org/10.1016/j.icarus.2012.11.039.

Hiroi T., Pieters C. M., Zolensky M. E., and Lipschutz M. E. 1993. Evidence of thermal metamorphism on the C, G, B, and F asteroids. Science 261:1016-1018. https://doi.org/10. 1126/science. 261.5124.1016.

Hiroi T., Pieters C. M., Zolensky M. E., and Lipschutz M. E. 1994. Possible thermal metamorphism on the C, G, B and $\mathrm{F}$ asteroids detected from their reflectance spectra in comparison with carbonaceous chondrites. Proceedings of the NIPR Symposium on Antarctic Meteorites 7:230-243.

Hiroi T., Pieters C. M., Zolensky M. E., and Prinz M. 1996. Reflectance spectra (UV-3 $\mu \mathrm{m}$ ) of heated Ivuna (CI) meteorite and newly identified thermally metamorphosed CM chondrites (abstract). 27th Lunar and Planetary Science Conference. p. 551.

Howard K. T., Benedix G. K., Bland P. A., and Cressey G. 2009. Modal mineralogy of CM2 chondrites by X-ray diffraction (PSD-XRD). Part 1: Total phyllosilicate abundance and the degree of aqueous alteration. Geochimica et Cosmochimica Acta 73:4576-4589. https:// doi.org/10.1016/j.gca.2009.04.038.

Howard K. T., Benedix G. K., Bland P. A., and Cressey G. 2011. Modal mineralogy of CM chondrites by X-ray diffraction (PSD-XRD): Part 2. Degree, nature and settings of aqueous alteration. Geochimica et Cosmochimica Acta 75:2735-2751. https://doi.org/10.1016/j.gca.2011.02.021.

Irving A. J., Kuehner S. M., RumbleIII D., Korotev R. L., and Clary S. 2009. Moapa Valley: A second non-Antarctic CM1 chondrite from Nevada, USA (abstract \#5372). Annual Meteoritical Society meeting 72.

Ivanova M. A., Lorenz C. A., Nazarov M. A., Brandstaetter F., Franchi I. A., Moroz L. V., Clayton R. N., and Bychkov A. Y. 2010. Dhofar 225 and Dhofar 735: Relationship to CM2 chondrites and metamorphosed carbonaceous chondrites, Belgica-7904 and Yamato-86720. Meteoritics \& Planetary Science 45:1108-1123. https://doi. org/10.1111/j.1945-5100.2010.01064.

Izawa M. R. M., Schäfer T., Pietrasz V. B., Cloutis E. A., Mann P., Nathues A., Mengel K., Schäfer M., Thangjam G. S., Hoffmann M., Tait K. T., and Applin D. M. 2016.
Effects of viewing geometry, aggregation state, and particle size on reflectance spectra of the Murchison CM2 chondrite deconvolved to Dawn FC band passes. Icarus 266:235-248. https://doi.org/10.1016/j.icarus.2015.10.029.

Jaumann R., Stephan K., Krohn K., Matz K.-D., Otto K., Neumann W., Kneissl T., Schmedemann N., Schroeder S., Tosi F., De Sanctis M. C., Preusker F., Buczkowski D., Capaccioni F., Carsenty U., Elgner S., dervon Gathen I., Gieber T., Hiesinger H., Hoffmann M., Kersten E., Li J.Y., McCord T. B., McFadden L., Mottola S., Nathues A., Neesemann A., Raymond C., Roatsch T., Russell C. T., Schmidt B., Schulzeck F., Wagner R., and Williams D. A. 2016. Age-dependent morphological and compositional variations on Ceres (abstract \#1455). 47th Lunar and Planetary Science Conference. CD-ROM.

Johnson T. V. and Fanale F. P. 1973. Optical properties of carbonaceous chondrites and their relationship to asteroids. Journal of Geophysical Research 78:8507-8518.

Johnson C. A. and Prinz M. 1993. Carbonate compositions in $\mathrm{CM}$ and $\mathrm{CI}$ chondrites and implications for aqueous alteration. Geochimica et Cosmochimica Acta 57:2843-2852. https://doi.org/10.1016/0016-7037(93)90393B.

King T. V. V. and Clark R. N. 1989. Spectral characteristics of chlorites and $\mathrm{Mg}$-serpentines using high resolution reflectance spectroscopy. Journal of Geophysical Research 94:13,997-14,008.

King T. V. V., Clark R. N., Calvin W. M., Sherman D. M., and Brown R. H. 1992. Evidence for ammonium-bearing minerals on Ceres. Science 255:1551-1553. https://doi.org/ 10.1126/science.255.5051.1551.

King A. J., Schofield P. F., Howard K. T., and Russell S. S. 2015. Modal mineralogy of CI and CI-like chondrites by X-ray diffraction. Geochimica et Cosmochimica Acta 165:148-160. https://doi.org/10.1016/j.gca.2015.05.038.

King A. J., Schofield P. F., and Russell S. S. 2017. Type 1 aqueous alteration in $\mathrm{CM}$ carbonaceous chondrites: Implications for the evolution of water-rich asteroids. Meteoritics \& Planetary Science 52:1197-1215. https://doi. org/10.1111/maps.12872.

Larson H. P., Feierberg M. A., Fink U., and Smith H. A. 1979. Remote spectroscopic identification of carbonaceous chondrite mineralogies: Applications to Ceres and Pallas. Icarus 39:257-271. https://doi.org/10.1016/0019-1035(79) 90168-4.

Lazarro D., Angeli C., Carvano J. M., Mothe-Diniz T., Duffard R., and Florczak M. 2004. S3OS2: The visible spectroscopic survey of 820 asteroids. Icarus 172:179-220. https://doi.org/10.1016/j.icarus.2004.06.006.

Lebofsky L. A. 1978. Asteroid 1 Ceres: Evidence for water of hydration. Monthly Notices of the Royal Astronomical Society 182:17-21.

Lebofsky L. A., Feierberg M. A., Tokunaga A. T., Larson H. P., and Johnson J. R. 1981. The 1.7- to 4.2- $\mu$ m spectrum of asteroid 1 Ceres: Evidence for structural water in clay minerals. Icarus 48:453-459. https://doi.org/10.1016/00191035(81)90055-5.

McAdam M. M., Sunshine J. M., Howard K. T., and McCoy T. M. 2015. Aqueous alteration on asteroids: Linking the mineralogy and spectroscopy of $\mathrm{CM}$ and $\mathrm{CI}$ chondrites. Icarus 245:320-332. https://doi.org/10.1016/j.icarus.2014. 09.041 .

McSween H. Y. 1979. Alteration in CM carbonaceous chondrites inferred from modal and chemical variations in 
matrix. Geochimica et Cosmochimica Acta 43:1761-1770. https://doi.org/10.1016/0016-7037(79)90024-3.

McSween H. Y. Jr., Emery J. P., Rivkin A. S., Toplis M. J., Castillo-Rogez J. C., Prettyman T. H., De Sanctis M. C., Pieters C. M., Raymond C. A., and Russell C. T. 2017. Carbonaceous chondrites as analogs for the composition and alteration of Ceres. Meteoritics \& Planetary Science. https://doi.org/10.1111/maps. 12947.

Milliken R. E. and Rivkin A. S. 2009. Brucite and carbonate assemblages from altered olivine-rich materials on Ceres. Nature Geoscience 2:258-261. https://doi.org/10.1038/nge o478.

Müller W. F., Kurat G., and Kracher A. 1979. Chemical and crystallographic study of cronstedtite in the matrix of the Cochabamb CM2 carbonaceous chondrite. Tschermaks Mineralogische und Petrographische Mitteilungen 26:293304.

Nathues A., Hoffmann M., Schaefer M., Le Corre L., Reddy V., Platz T., Cloutis E. A., Christensen U., Kneissl T., Li J.-Y., Mengel K., Schmedemann N., Schaefer T., Russell C. T., Applin D. M., Buczkowski D. L., Izawa M. R. M., Keller H. U., O'Brien D. P., Pieters C. M., Raymond C. A., Ripken J., Schenk P. M., Schmidt B. E., Sierks H., Sykes M. V., Thangjam G. S., and Vincent J.-B. 2015. Sublimation in bright spots on (1) Ceres. Nature 528:237240. https://doi.org/10.1038/nature15754.

Neveu M., Desch S. J., and Castillo-Rogez J. C. 2017. Aqueous geochemistry in icy world interiors: Equilibrium fluid, rock, and gas compositions, and fate of antifreezes and radionuclides. Geochimica et Cosmochimica Acta 212:324-371. https://doi.org/10.1016/j.gca.2017.06.023.

Perna D., Kaňuchová Z., Ieva S., Fornasier S., Barucci M. A., Lantz C., Dotto E., and Strazzulla G. 2015. Short-term variability on the surface of (1) Ceres. Astronomy \& Astrophysics 575:L1. https://doi.org/10.1051/0004-6361/ 201425304.

Pieters C. M., Thangiam G. S., Nathues A., Hoffman M., Platz T., De Sanctis M. C., Ammannito E., Tosi F., Zambon F., Pasckert J. H., Hiesinger H., Schröder S. E., Jaumann R., Matz K.-D., Castillo-Rogez J. C., Ruesch O., McFadden L. A., O'Brien D. P., Sykes M., Raymond C. A., and Russell C. T. 2017. Geologic constraints on the origin of red organic-rich material on Ceres. Meteoritics \& Planetary Science. https://doi.org/10.1111/maps.13008.

Prettyman T. H., Yamashita N., Toplis M. J., McSween H. Y., Schörghofer N., Marchi S., Feldman W. C., CastilloRogez J. C., Forni O., Lawrence D. J., Ammannito E., Ehlmann B. L., Sizemore H. G., Joy S. P., Polanskey C. A., Rayman M. D., Raymond C. A., and Russell C. T. 2017. Extensive water ice within Ceres' aqueously altered regolith: Evidence from nuclear spectroscopy. Science 355:55-59. https://doi.org/10.1126/science.aah6765.

Preusker F., Scholten F., Matz K.-D., Elgner S., Jaumann R., Roatsch T., Joy S. P., Polanskey C. A., Raymond C. A., and Russell C. T. 2016. Dawn at Ceres-Shape model and rotational state (abstract \#1954). 47th Lunar and Planetary Science Conference. CD-ROM.

Roatsch T., Kersten E., Matz K.-D., Preusker F., Scholten F., Jaumann R., Raymond C. A., and Russell C. T. 2015. Ceres survey atlas derived from Dawn Framing Camera images. Planetary and Space Science 121:115-120. https://d oi.org/10.1016/j.pss.2015.12.005.

Roatsch T., Kersten E., Matz K.-D., Preusker F., Scholten F., Jaumann R., Raymond C. A., and Russell C. T. 2016.
High-resolution Ceres high altitude mapping orbit atlas derived from Dawn framing camera images. Planetary and Space Science 129:103-107. https://doi.org/10.1016/j.pss. 2016.05.011.

Rubin A. E. 2012. Collisional facilitation of aqueous alteration of $\mathrm{CM}$ and $\mathrm{CV}$ carbonaceous chondrites. Geochimica et Cosmochimica Acta 90:181-194. https://doi. org/10.1016/j.gca.2012.05.016.

Rubin A. E., Trigo-Rodríguez J. M., Huber H., and Wasson J. T. 2007. Progressive aqueous alteration of CM carbonaceous chondrites. Geochimica et Cosmochimica Acta 71:2361-2382. https://doi.org/10.1016/j.gca.2007.02. 008.

Schäfer T., Nathues A., Mengel K., Izawa M. R. M., Cloutis E. A., Schäfer M., and Hoffmann M. 2016. Spectral parameters for Dawn FC color data: Carbonaceous chondrites and aqueous alteration products as potential cerean analog materials. Icarus 265:149-160. https://doi. org/10.1016/j.icarus.2015.10.005.

Schmedemann N., Kneissl T., Neesemann A., Stephan K., Jaumann R., Krohn K., Michael G. G., Matz K. D., Otto K., Raymond C. A., and Russell C. T. 2016. Timing of optical maturation of recently exposed material on Ceres. Geophysical Research Letters 43:11,987-11,993. https://doi. org/10.1002/2016GL071143.

Schröder S. E., Maue T., Gutiérrez Marqués P., Mottola S., Aye K. M., Sierks H., Keller H. U., and Nathues A. 2013. In-flight calibration of the Dawn Framing Camera. Icarus 226:1304-1317. https://doi.org/10.1016/j.icarus.2013.07.036.

Schröder S. E., Mottola S., Matz K.-D., and Roatsch T. 2014. In-flight calibration of the Dawn Framing Camera II: Flat fields and stray light correction. Icarus 234:99-108. https://doi.org/10.1016/j.icarus.2014.02.018.

Schröder S. E., Mottola S., Carsenty U., Ciarniello M., Jaumann R., Li J.-Y., Longobardo A., Palmer E., Pieters C., Preusker F., Raymond C. A., and Russell C. T. 2017. Resolved spectrophotometric properties of the Ceres surface from Dawn Framing Camera images. Icarus 288:201-225. https://doi.org/10.1016/j.icarus.2017.01.026.

Sierks H., Keller H. U., Jaumann R., Michalik H., Behnke T., Bubenhagen F., Büttner I., Carsenty U., Christensen U., Enge R., Fiethe B., Gutiérrez Marqués P., Hartwig H., Krüger H., Kühne W., Maue T., Mottola S., Nathues A., Reiche K.-U., Richards M. L., Roatsch T., derSchrö S. E., Szemerey I., and Tschentscher M. 2011. The Dawn Framing Camera. In The Dawn mission to minor planets 4 Vesta and 1 Ceres, edited by Russell C. T. and Raymond C. A. New York: Springer. pp. 263-327.

Stephan K., Jaumann R., Krohn K., Schmedemann N., Zambon F., Tosi F., Carrozzo F. G., McFadden L. A., Otto K., De Sanctis M. C., Ammannito E., Matz K.-D., Roatsch T., Preusker F., Raymond C. A., and Russell C. T. 2017. An investigation of the bluish material on Ceres. Geophysical Research Letters 44:1660-1668. https://doi.org/ 10.1002/2016GL071652.

Takir D., Emery J. P., McSween H. Y., Hibbitts C. A., Clark R. N., Pearson N., and Wang A. 2013. Nature and degree of aqueous alteration in CM and CI carbonaceous chondrites. Meteoritics \& Planetary Science 48:1618-1637. https://doi.org/10.1111/maps.12171.

Tomeoka K. 1990. Matrix compositions and mineralogy of the Alais and Ivuna CI carbonaceous chondrites (abstract \#1256). 21st Lunar and Planetary Science Conference. CDROM. 
Tomeoka K. and Buseck P. R. 1985. Indicators of aqueous alteration in $\mathrm{CM}$ carbonaceous chondrites: Microtextures of a layered mineral containing $\mathrm{Fe}, \mathrm{S}, \mathrm{O}$ and $\mathrm{Ni}$. Geochimica et Cosmochimica Acta 49:2149-2163.

Tomeoka K. and Buseck P. R. 1988. Matrix mineralogy of the Orgueil CI carbonaceous chondrite. Geochimica et Cosmochimica Acta 52:1627-1640. https://doi.org/10.1016/ 0016-7037(88)90231-1.

Tomeoka K., Kojima H., and Keizo Y. 1989. Yamato-82162: A new kind of CI carbonaceous chondrite in Antarctica. Proceedings of the NIPR Symposium on Antarctic Meteorites 2:36-54.

Vernazza P., Mothé-Diniz T., Barucci M. A., Birlan M., Carvano J. M., Strazzulla G., Fulchignoni M., and Migliorini A. 2005. Analysis of near-IR spectra of 1 Ceres and 4 Vesta, targets of the Dawn mission. Astronomy \& Astrophysics 436:1113-1121. https://doi.org/10.1051/0004-6361:20042506.

\section{SUPPORTING INFORMATION}

Additional supporting information may be found in the online version of this article:

Appendix S1. Additional corrections applied to Framing Camera I/F data.
Vilas F. and McFadden L. A. 1992. CCD reflectance spectra of selected asteroids. Icarus 100:85-94. https://doi.org/10. 1016/0019-1035(92)90020-8.

Vilas F., Larson S. M., Hatch E. C., and Jarvis K. S. 1993. CCD reflectance spectra of selected asteroids. II. Lowalbedo asteroid spectra and data extraction techniques. Icarus 105:67-78. https://doi.org/10.1006/icar.1993.1111.

Zolensky M., Barrett R., and Browning L. 1993. Mineralogy and composition of matrix and chondrule rims in carbonaceous chondrites. Geochimica et Cosmochimica Acta 57:3123-3148. https://doi.org/10.1016/0016-7037(93)90298-B.

Zolensky M. E., Mittlefehldt D. W., Lipschutz M. E., Wang M.-S., Clayton R. N., Mayeda T. K., Grady M. M., and Pillinger C. B. D. 1997. CM chondrites exhibit the complete petrologic range from type 2 to 1 . Geochimica et Cosmochimica Acta 61:5099-5115. https://doi.org/10.1016/ S0016-7037(97)00357-8.

Appendix S2. Exclusion of FC filter F2 at $0.555 \mu \mathrm{m}$. Appendix S3. Histograms of the slope and reflectance values on Ceres.

Appendix S4. CC discrimination scheme.

Appendix S5. Table of meteorites used for CC discrimination scheme. 